International Journal of Quantum Information

Vol. 15, No. 7 (2017) 1750054 (41 pages)

(C) The Author(s)

DOI: $10.1142 / \mathrm{S} 021974991750054 \mathrm{X}$

\title{
Nongeneric positive partial transpose states of rank five in $3 \times 3$ dimensions
}

\author{
Leif Ove Hansen and Jan Myrheim* \\ Department of Physics, \\ Norwegian University of Science and Technology, \\ N-7491 Trondheim, Norway \\ *jan.myrheim@ntnu.no
}

Received 14 September 2017

Accepted 15 October 2017

Published 4 December 2017

In $3 \times 3$ dimensions, entangled mixed states that are positive under partial transposition (PPT states) must have rank at least four. These rank four states are completely understood. We say that they have rank $(4,4)$ since both a state $\rho$ and its partial transpose $\rho^{P}$ have rank four. The next problem is to understand the extremal PPT states of rank $(5,5)$. We call two states $\mathrm{SL} \otimes \mathrm{SL}$-equivalent if they are related by a product transformation. A generic rank $(5,5)$ PPT state $\rho$ is extremal, and both $\rho$ and $\rho^{P}$ have six product vectors in their ranges, and no product vectors in their kernels. The three numbers $\{6,6 ; 0\}$ are SL $\otimes$ SL-invariants that help us classify the state. There is no analytical understanding of such states. We have studied numerically a few types of nongeneric rank five PPT states, in particular, states with one or more product vectors in their kernels. We find an interesting new analytical construction of all rank four extremal PPT states, up to SL $\otimes$ SL-equivalence, where they appear as boundary states on one single five-dimensional face on the set of normalized PPT states. The interior of the face consists of rank $(5,5)$ states with four common product vectors in their kernels, it is a simplex of separable states surrounded by entangled PPT states. We say that a state $\rho$ is $\mathrm{SL} \otimes \mathrm{SL}$-symmetric if $\rho$ and $\rho^{P}$ are $\mathrm{SL} \otimes \mathrm{SL}$-equivalent, and is genuinely $\mathrm{SL} \otimes \mathrm{SL}$-symmetric if it is $\mathrm{SL} \otimes \mathrm{SL}$-equivalent to a state $\tau$ with $\tau=\tau^{P}$. Genuine SL $\otimes$ SL-symmetry implies a special form of SL $\otimes$ SL-symmetry. We have produced numerically, by a special method, a random sample of rank $(5,5) \mathrm{SL} \otimes \mathrm{SL}$-symmetric states. About 50 of these are of type $\{6,6 ; 0\}$, among those all are extremal and about half are genuinely $\mathrm{SL} \otimes \mathrm{SL}$-symmetric. All these genuinely $\mathrm{SL} \otimes \mathrm{SL}$-symmetric states can be transformed to have a circulant form. We find however that this is not a generic property of genuinely $\mathrm{SL} \otimes \mathrm{SL}$-symmetric states. The remaining $\mathrm{SL} \otimes \mathrm{SL}$-symmetric states found in the search have product vectors in their kernels, and they inspired us to study such states without regard to SL $\otimes$ SL-symmetry.

Keywords: Bound entanglement; low-rank entangled states.

\footnotetext{
${ }^{*}$ Corresponding author.

This is an Open Access article published by World Scientific Publishing Company. It is distributed under the terms of the Creative Commons Attribution 4.0 (CC-BY) License. Further distribution of this work is permitted, provided the original work is properly cited.
} 


\section{Introduction}

Entanglement between subsystems of a composite quantum system is a phenomenon which has no counterpart in classical physics. Entangled quantum states show correlations in measurements which cannot be modeled within any local theory, including classical physics. A classical local description of such systems implies the so-called Bell inequalities, ${ }^{1}$ which are violated in experiments. ${ }^{2}$ So, entangled quantum states exhibit a nonlocality that excludes any local theory though nonlocal deterministic theories are still possible.

Pure product states are the only pure quantum states that are not entangled, and they resemble pure classical states in that they have no correlations at all. By definition, a mixed quantum state is a statistical ensemble of pure quantum states, and it is represented mathematically by a density matrix. One single density matrix may represent many different ensembles. A basic postulate is that there is no way to distinguish experimentally between different ensembles represented by the same density matrix.

A mixed quantum state is said to be separable if it can be mixed entirely from pure product states. The entangled states are exactly those that are not separable. While the separability problem for pure states is solved entirely via Schmidt decomposition of state vectors, the problem of how to characterize the set $\mathcal{S}$ of separable mixed states, and to decide whether a given mixed state is separable or entangled, is known to be a very difficult mathematical problem in general, and it has been demonstrated that operational procedures are NP-hard. ${ }^{3}$

In recent years, these problems have been given considerable attention, mainly due to the fact that quantum entanglement has found use in many applications. Many new developments require an understanding of entanglement as a resource, in areas such as quantum communication, quantum cryptography and quantum computing. How to prepare, manipulate and detect entangled quantum states has become an important issue.

Sound operational methods or criteria for entanglement checking only exist for special cases and/or for low-dimensional systems. The separable states have the property that they remain positive after partial transposition. We call them PPT states. The set $\mathcal{P}$ of PPT states is in general larger than the set $\mathcal{S}$ of separable states, but the difference between the two sets is small in low dimensions, and in the $2 \times 2$ and $2 \times 3$ systems, $\mathcal{P}=\mathcal{S} .{ }^{4}$ Thus, the condition of PPT, known as the Peres separability criterion, ${ }^{5}$ is completely adequate as long as the system has dimension $N=N_{A} N_{B} \leq 6$.

For systems of dimension $N=N_{A} N_{B} \geq 8$, entangled PPT states, also known as states with bound entanglement, exist. For these states, that make up the difference between $\mathcal{P}$ and $\mathcal{S}$, the Peres criterion is not sufficient.

Another useful criterion is the range criterion, which states that if a state is separable, then its range is spanned by a set of product vectors $w_{i}=u_{i} \otimes v_{i}$ such that the range of the partial transpose $\rho^{P}$ is spanned by $\tilde{w}_{i}=u_{i} \otimes v_{i}^{*}$. It was shown that 
this criterion is independent from the PPT criterion, as there are PPT entangled states that violate the range criterion and there are non-PPT states satisfying it. ${ }^{6}$

The close relation between PPT states and product vectors has been used to prove the separability of sufficiently low rank PPT states. It was shown that all PPT states of rank at most $N_{B}$ supported on a Hilbert space of dimension $N=N_{A} N_{B}$ with $N_{A} \leq N_{B}$ are separable. ${ }^{7}$

For the $3 \times 3$ system, this means that the lowest possible rank of an entangled PPT state is four. Bennett et al. ${ }^{8,9}$ introduced a method for constructing low rank entangled PPT states by using unextendible product bases (UPBs). A UPB is defined as a maximal set of orthogonal product vectors which is not a complete basis of the Hilbert space. If one constructs an orthogonal projection $Q$ onto the UPB, then its complementary projection $P=I-Q$ is an entangled PPT state. The UPB construction is most successful in the case of rank four PPT states in the $3 \times 3$ system, where it leads to a construction of all entangled PPT states of rank four. ${ }^{10,11}$

This UPB strategy fails for rank five states in the $3 \times 3$ system, since a UPB must lie in the kernel of the state it defines, and no UPB has only four elements. The lack of such a simple construction method makes the characterization of entangled PPT states in the $3 \times 3$ system a much more challenging problem for the rank five states than for the rank four states. We report here a study of the rank five states, describing in particular several nongeneric forms.

A PPT state is partly characterized by two numbers $(m, n)$, where $m$ is its rank and $n$ is the rank of its partial transpose. A necessary condition for a PPT state in dimension $3 \times 3$ to be extremal is that $m^{2}+n^{2} \leq 82 .{ }^{12}$ Most of the states we have studied have rank $(5,5)$, but it should be noted that states of rank $(5,6)$ and rank $(5,7)$ are also easy to find numerically, and they are generically extremal. There also exist PPT states of rank $(5,8)$, but they can not be extremal. ${ }^{13}$

\section{Outline of the paper}

The contents of the present paper are organized in the following manner.

In Secs. 2 and 3, we review some linear algebra and introduce notation. We emphasize the importance of product vectors and product transformations in our study of low rank PPT states in bipartite composite systems. It is useful to classify such a state by the number of product vectors in its range and kernel.

Since a generic subspace of dimension four contains no product vectors, a rank five state with product vectors in its kernel is nongeneric. In Sec. 4, we define a standard form for PPT states of rank five with four product vectors in the kernel. All these special PPT states of rank $(5,5)$ are nonextremal, but this scheme leads to a new method for constructing extremal PPT states of rank $(4,4)$, and is highly relevant to our further study of nongeneric PPT states of rank $(5,5)$, presented in Sec. 7.

In Sec. 5, we review some features regarding product vectors in generic subspaces of dimension five in the $3 \times 3$ system. We also discuss nongeneric subspaces in the 
$3 \times 3$ system, specifically how to construct pairs of orthogonal subspaces $\mathcal{U}$ and $\mathcal{V}$ with $|\mathcal{U}|=5$ and $|\mathcal{V}|=4$, such that the number of product vectors in $\mathcal{V}$ is nonzero.

In Sec. 6, a summary of our numerical results on rank five PPT states is presented. This includes data for the generic states $\rho$ with no product vectors in $\operatorname{Ker} \rho$, and several nongeneric cases with up to four product vectors in Ker $\rho$. We also present some results from our random searches for SL $\otimes$ SL-symmetric states.

In Sec. 7, we present a collection of nongeneric standard forms for orthogonal subspaces $\mathcal{U}$ and $\mathcal{V}$ with dimensions five and four, respectively. The nongeneric feature is that the number of product vectors in $\mathcal{V}$ is nonzero. For the various $\mathcal{U}$ and $\mathcal{V}$, we have produced $\operatorname{PPT}$ states of $\operatorname{rank}(5,5)$ with $\operatorname{Img} \rho=\mathcal{U}$ and $\operatorname{Ker} \rho=\mathcal{V}$. The number of product vectors in $\operatorname{Img} \rho$ is either six or infinite, while the number of product vectors in Ker $\rho$ ranges from one to four. The latter is presented as a special case in Sec. 4.

Finally, in Sec. 8, we present a very special five-dimensional subspace of $\mathbb{C}^{9}$ that contains only two product vectors with one product vector in the orthogonal complement. We construct analytically a set of states found by transformation to a standard form of one particular rank $(5,5)$ nonextremal PPT state in this subspace. This nonextremal PPT state was found completely by chance in our random searches for $\mathrm{SL} \otimes \mathrm{SL}$-symmetric states.

\section{Basic Linear Algebra}

We want to review some basic concepts of linear algebra, partly in order to define our notation and make the paper self-contained, and partly in order to review some useful but less well-known facts.

\subsection{Density matrices}

The natural structure of the set $H_{N}$ of Hermitian $N \times N$ matrices is that of a real Hilbert space of dimension $N^{2}$ with the scalar product

$$
(X, Y)=\operatorname{Tr}(X Y)
$$

The set of mixed states, or density matrices, is defined as

$$
\mathcal{D}=\mathcal{D}_{N}=\left\{\rho \in H_{N} \mid \rho \geq 0, \operatorname{Tr} \rho=1\right\}
$$

A density matrix $\rho$ has a spectral representation in terms of a complete set of orthonormal eigenvectors $\psi_{i} \in \mathbb{C}^{N}$ with eigenvalues $\lambda_{i} \geq 0$,

$$
\rho=\sum_{i=1}^{N} \lambda_{i} \psi_{i} \psi_{i}^{\dagger}, \quad \psi_{i}^{\dagger} \psi_{j}=\delta_{i j}, \quad \sum_{i=1}^{N} \psi_{i} \psi_{i}^{\dagger}=I
$$


The spectral representation is one particular ensemble representation of $\rho$. It implies that

$$
\operatorname{Tr} \rho=\sum_{i=1}^{N} \lambda_{i} .
$$

The rank of $\rho$ is the number of eigenvalues $\lambda_{i}>0$. The matrices

$$
P=\sum_{i, \lambda_{i}>0} \psi_{i} \psi_{i}^{\dagger}, \quad Q=I-P=\sum_{i, \lambda_{i}=0} \psi_{i} \psi_{i}^{\dagger}
$$

are Hermitian and project orthogonally onto the two complementary orthogonal subspaces Img $\rho$, the range of $\rho$, and $\operatorname{Ker} \rho$, the kernel (or null space) of $\rho$.

When all $\lambda_{i} \geq 0$, we say that $\rho$ is positive (or positive semidefinite) and write $\rho \geq 0$. An equivalent condition is that $\psi^{\dagger} \rho \psi \geq 0$ for all $\psi \in \mathbb{C}^{N}$. It follows from the last inequality and the spectral representation of $\rho$ that $\psi^{\dagger} \rho \psi=0 \Longleftrightarrow \rho \psi=0$.

The fact that the positivity conditions $\psi^{\dagger} \rho \psi \geq 0$ are linear in $\rho$ implies that $\mathcal{D}$ is a convex set, so that if $\rho$ is a proper convex combination of $\rho_{1}, \rho_{2} \in \mathcal{D}$,

$$
\rho=p \rho_{1}+(1-p) \rho_{2}, \quad 0<p<1,
$$

then $\rho \in \mathcal{D}$. Furthermore, since

$$
\text { Ker } \rho=\{\psi \mid \rho \psi=0\}=\left\{\psi \mid \psi^{\dagger} \rho \psi=0\right\},
$$

when $\rho \geq 0$, it follows that

$$
\operatorname{Ker} \rho=\operatorname{Ker} \rho_{1} \cap \operatorname{Ker} \rho_{2}
$$

is independent of $p$, when $\rho$ is a proper convex combination as above. Since Ker $\rho$ is independent of $p$, so is $\operatorname{Img} \rho=(\operatorname{Ker} \rho)^{\perp}$.

A compact (closed and bounded) convex set is determined by its extremal points, those points that are not convex combinations of other points in the set. The extremal points of $\mathcal{D}$ are the pure states of the form $\rho=\psi \psi^{\dagger}$ with $\psi \in \mathbb{C}^{N}$ and $\psi^{\dagger} \psi=1$. Thus, the spectral representation is an expansion of $\rho$ as a convex combination of $m$ extremal points in $\mathcal{D}$ where $m$ is the rank of $\rho$.

\subsection{Perturbations and extremality in $\mathcal{D}$}

Let $\rho$ be a density matrix and define the projections $P$ and $Q$ as in Eq. (5). Consider a perturbation of the form

$$
\rho \rightarrow \rho^{\prime}=\rho+\epsilon A
$$

where $A \neq 0$ is Hermitian, and $\operatorname{Tr} A=0$ so that $\operatorname{Tr} \rho^{\prime}=\operatorname{Tr} \rho$. The real parameter $\epsilon$ may be infinitesimal or finite.

We observe that if $\operatorname{Img} A \subset \operatorname{Img} \rho$, or equivalently if $P A P=A$, then there will be a finite range of values of $\epsilon$, say $\epsilon_{1} \leq \epsilon \leq \epsilon_{2}$ with $\epsilon_{1}<0<\epsilon_{2}$, such that $\rho^{\prime} \in \mathcal{D}$ and 
$\operatorname{Img} \rho=\operatorname{Img} \rho^{\prime}$. This is so because the eigenvectors of $\rho$ with zero eigenvalue will remain eigenvectors of $\rho^{\prime}$ with zero eigenvalue, and all the positive eigenvalues of $\rho$ will change continuously with $\epsilon$ into eigenvalues of $\rho^{\prime}$. Since $\mathcal{D}$ is compact, we may choose $\epsilon_{1}$ and $\epsilon_{2}$ such that $\rho^{\prime}$ has at least one negative eigenvalue when either $\epsilon<\epsilon_{1}$ or $\epsilon>\epsilon_{2}$. The negative eigenvalue becomes zero at $\epsilon=\epsilon_{1}$ or $\epsilon=\epsilon_{2}$, this makes Ker $\rho^{\prime}$ strictly larger than Ker $\rho$ and $\operatorname{Img} \rho^{\prime}$ strictly smaller than $\operatorname{Img} \rho$ in both limits $\epsilon=\epsilon_{1}$ and $\epsilon=\epsilon_{2}$.

The other way around, if $\rho^{\prime} \in \mathcal{D}$ for $\epsilon_{1} \leq \epsilon \leq \epsilon_{2}$ with $\epsilon_{1}<0<\epsilon_{2}$, then $\rho^{\prime}$ is a convex combination of $\rho+\epsilon_{1} A$ and $\rho+\epsilon_{2} A$ for every $\epsilon$ in the open interval $\epsilon_{1}<\epsilon<\epsilon_{2}$. Hence, Img $\rho^{\prime}$ is independent of $\epsilon$ in this open interval, implying that $\operatorname{Img} A \subseteq \operatorname{Img} \rho$ and $P A P=A$.

This gives us three equivalent formulations for the extremality condition of $\rho$ on $\mathcal{D}$. The state $\rho$ is extremal in $\mathcal{D}$ if and only if

- There exists no $A \neq 0$ with $\operatorname{Tr} A=0$ and $P A P=A$.

- The equation $P A P=A$ for the Hermitian matrix $A$ has $A=\rho$ as its only solution (up to proportionality).

- There exists no $\rho^{\prime} \in \mathcal{D}$ with $\rho^{\prime} \neq \rho$ and $\operatorname{Img} \rho^{\prime}=\operatorname{Img} \rho$.

We may replace the condition $P A P=A$ by the weaker condition $Q A Q=0$. By standard perturbation theory, it implies that the zero eigenvalues of $\rho$ do not change to first order in $\epsilon$. Thus, the perturbation in Eq. (9), with $Q A Q=0$ and $\epsilon$ infinitesimal, preserves the rank but not necessarily the range of $\rho$.

\subsection{Projection operators on $\boldsymbol{H}_{N}$}

Using the projections $P$ and $Q$ defined above, we can define projection operators $\mathbf{P}$, $\mathbf{Q}$ and $\mathbf{R}$ on $H_{N}$, the real Hilbert space of Hermitian $N \times N$ matrices, as follows:

$$
\begin{aligned}
& \mathbf{P} X=P X P, \\
& \mathbf{Q} X=Q X Q, \\
& \mathbf{R} X=(\mathbf{I}-\mathbf{P}-\mathbf{Q}) X .
\end{aligned}
$$

Here, $\mathbf{I}$ is the identity operator on $H_{N}$. It is straightforward to verify that these are complementary projections, with $\mathbf{P}^{2}=\mathbf{P}, \mathbf{Q}^{2}=\mathbf{Q}, \mathbf{P Q}=\mathbf{Q P}=\mathbf{0}$, and so on. They are symmetric with respect to the natural scalar product on $H_{N}$, hence they project orthogonally, and relative to an orthonormal basis for $H_{N}$, they are represented by symmetric matrices.

Relative to an orthonormal basis of $\mathbb{C}^{N}$ with the first $m$ basis vectors in Img $\rho$ and the last $k=N-m$ basis vectors in $\operatorname{Ker} \rho$, a Hermitian matrix $X$ takes the block form

$$
X=\left(\begin{array}{cc}
U & V \\
V^{\dagger} & W
\end{array}\right)
$$


with $U \in H_{m}$ and $W \in H_{k}$. In this basis, we have

$$
P=\left(\begin{array}{cc}
I_{m} & 0 \\
0 & 0
\end{array}\right), \quad Q=\left(\begin{array}{cc}
0 & 0 \\
0 & I_{k}
\end{array}\right)
$$

and hence

$$
\mathbf{P} X=\left(\begin{array}{ll}
U & 0 \\
0 & 0
\end{array}\right), \quad \mathbf{Q} X=\left(\begin{array}{cc}
0 & 0 \\
0 & W
\end{array}\right), \quad \mathbf{R} X=\left(\begin{array}{cc}
0 & V \\
V^{\dagger} & 0
\end{array}\right)
$$

\section{Composite Systems}

In order to describe entanglement in quantum systems, it is necessary to develop the basic theory of tensor product spaces. We do this for a bipartite system consisting of two subsystems A and B.

\subsection{Product vectors}

If $N=N_{A} N_{B}$, then the tensor product spaces $\mathbb{C}^{N}=\mathbb{C}^{N_{A}} \otimes \mathbb{C}^{N_{B}}$ (a complex tensor product) and $H_{N}=H_{N_{A}} \otimes H_{N_{B}}$ (a real tensor product) describe a composite quantum system with two subsystems $\mathrm{A}$ and $\mathrm{B}$ of Hilbert space dimensions $N_{A}$ and $N_{B}$.

A vector $\psi \in \mathbb{C}^{N}$ then has components $\psi_{I}=\psi_{i j}$, where

$$
I=1, \ldots, N \leftrightarrow i j=11,12, \ldots, 1 N_{B}, 21,22, \ldots, N_{A} N_{B}
$$

A product vector $\psi=\phi \otimes \chi$ has components $\psi_{i j}=\phi_{i} \chi_{j}$. We see that $\psi$ is a product vector if and only if its components satisfy the quadratic equations

$$
\psi_{i j} \psi_{k l}-\psi_{i l} \psi_{k j}=0 \text {. }
$$

All these equations are not independent, the number of independent complex equations is

$$
K=\left(N_{A}-1\right)\left(N_{B}-1\right)=N-N_{A}-N_{B}+1 .
$$

For example, if $\psi_{1} \neq 0$, we get a complete set of independent equations by taking $i=j=1, k=2, \ldots, N_{A}$ and $l=2, \ldots, N_{B}$.

Since Eq. (15) is homogeneous, any solution $\psi \neq 0$ gives rise to a one-parameter family of solutions $c \psi$, where $c \in \mathbb{C}$. A vector $\psi$ in a subspace of dimension $n$ has $n$ independent complex components. Since the most general nonzero solution must contain at least one free complex parameter, we conclude that a generic subspace of dimension $n$ will contain nonzero product vectors if and only if

$$
n \geq K+1 \text {. }
$$

The limiting dimension

$$
n=K+1=N-N_{A}-N_{B}+2
$$


is particularly interesting. In this special case, a nonzero solution will contain exactly one free parameter, which has to be a complex normalization constant. Thus, up to proportionality, there will exist a finite set of product vectors in a generic subspace of this dimension, in fact the number of product vectors is ${ }^{14}$

$$
p=\left(\begin{array}{c}
N_{A}+N_{B}-2 \\
N_{A}-1
\end{array}\right)=\frac{\left(N_{A}+N_{B}-2\right) !}{\left(N_{A}-1\right) !\left(N_{B}-1\right) !} .
$$

A generic subspace of lower dimension will contain no nonzero product vector, whereas any subspace of higher dimension will contain a continuous infinity of different product vectors (different in the sense that they are not proportional).

These results hold for generic subspaces. It is trivially clear that nongeneric subspaces with low dimensions exist that contain product vectors. In the special case $N_{A}=N_{B}=3$ studied here, the limiting dimension given by Eq. (18) is five, and the number of product vectors given by Eq. (19) is six. As described in Sec. 8, we have found one special example of a five-dimensional subspace with only two product vectors. Nongeneric cases are treated more generally in Ref. 11.

The facts that product vectors always exist in $K+1$ and higher dimensions, but not always in $K$ and lower dimensions, are special cases of a theorem proved by Parthasarathy for systems composed of any number of subsystems. ${ }^{15}$ These results have profound implications for the construction of UPBs.

\subsection{Partial transposition and separability}

The following relation between matrix elements

$$
\left(X^{P}\right)_{i j ; k l}=X_{i l ; k j}
$$

defines the partial transpose $X^{P}=X^{T_{B}}$ of the matrix $X$ with respect to the second subsystem B. The partial transpose with respect to the first subsystem $A$ is $X^{T_{A}}=X^{P T}$, where $T$ denotes total transposition. Since we work with Hermitian matrices, $T$ is the same as complex conjugation. The partial transposition is transposition of the individual submatrices of dimension $N_{B} \times N_{B}$ in the $N \times N$ matrix $X$. If $X=Y \otimes Z$, then $X^{P}=Y \otimes Z^{T}$.

A density matrix $\rho$ is called separable if it is a convex combination of tensor product pure states,

$$
\rho=\sum_{k} p_{k} w_{k} w_{k}^{\dagger}
$$

with $w_{k}=u_{k} \otimes v_{k} \in \mathbb{C}^{N}, p_{k}>0$, and $\sum_{k} p_{k}=1$. It follows that

$$
\rho^{P}=\sum_{k} p_{k}\left(u_{k} u_{k}^{\dagger}\right) \otimes\left(v_{k} v_{k}^{\dagger}\right)^{T}=\sum_{k} p_{k}\left(u_{k} u_{k}^{\dagger}\right) \otimes\left(v_{k}^{*} v_{k}^{T}\right) .
$$

We denote the set of separable density matrices by $\mathcal{S}$. 
The obvious fact that $\rho^{P}$ is positive when $\rho$ is separable is known as the Peres criterion, it is an easily testable necessary condition for separability. For this reason, it is of interest to study the set of PPT matrices, defined as

$$
\mathcal{P}=\left\{\rho \in \mathcal{D} \mid \rho^{P} \geq 0\right\}=\mathcal{D} \cap \mathcal{D}^{P}
$$

We may call it the Peres set. A well-known result is that $\mathcal{P}=\mathcal{S}$ for $N=N_{A} N_{B} \leq 6$, whereas $\mathcal{P}$ is strictly larger than $\mathcal{S}$ in higher dimensions. ${ }^{4}$

We will classify low rank PPT states by the ranks $(m, n)$ of $\rho$ and $\rho^{P}$, respectively. Here, we study the special case $N_{A}=N_{B}=3$, then the ranks $(m, n)$ and $(n, m)$ are equivalent for the purpose of classification, because of the symmetric roles of the subsystems A and B.

\subsection{Product vectors in the kernel and range}

Recall that $\psi^{\dagger} \rho \psi=0 \Longleftrightarrow \rho \psi=0$ when $\rho \geq 0$, and similarly for $\rho^{P}$. The identity

$$
(x \otimes y)^{\dagger} \rho(x \otimes y)=\left(x \otimes y^{*}\right)^{\dagger} \rho^{P}\left(x \otimes y^{*}\right)
$$

therefore implies, for a PPT state $\rho$, that $x \otimes y \in \operatorname{Ker} \rho$ if and only if $x \otimes y^{*} \in \operatorname{Ker} \rho^{P}$.

Let the number of product vectors in $\operatorname{Img} \rho, \operatorname{Img} \rho^{P}$ and Ker $\rho$ be respectively $n_{\text {img }}$, $\tilde{n}_{\text {img }}$ and $n_{\text {ker }}$. Then, $n_{\text {ker }}$ is also the number of product vectors in $\operatorname{Ker} \rho^{P}$, and $\rho$ is characterized by $\left\{n_{\text {img }}, \tilde{n}_{\text {img }} ; n_{\text {ker }}\right\}$. We find numerically that the generic entangled PPT states of rank $(5,5)$ are $\{6,6 ; 0\}$ states.

We write the product vectors in $\operatorname{Img} \rho$ as

$$
w_{i}=u_{i} \otimes v_{i}, \quad i=1, \ldots, n_{\mathrm{img}} .
$$

And likewise for $\operatorname{Ker} \rho$,

$$
z_{j}=x_{j} \otimes y_{j}, \quad j=1, \ldots, n_{\mathrm{ker}}
$$

Since the two subspaces are orthogonal, it is necessary that $w_{i}^{\dagger} z_{j}=0$ for all $i, j$, hence for every pair $i, j$, we must have either $u_{i}^{\dagger} x_{j}=0$ or $v_{i}^{\dagger} y_{j}=0$.

\subsection{The range criterion and edge states}

If $\rho$ is the separable state given in Eq. (21), then $\operatorname{Img} \rho$ is spanned by the product vectors $w_{k}=u_{k} \otimes v_{k}$, and $\operatorname{Img} \rho^{P}$ is spanned by the partially conjugated product vectors $\tilde{w}_{k}=u_{k} \otimes v_{k}^{*}$. No such relation is known to exist between product vectors in $\operatorname{Img} \rho$ and $\operatorname{Img} \rho^{P}$ when $\rho$ is an entangled PPT state.

The existence of a set of product vectors spanning the range of $\rho$, such that the partially conjugated product vectors span the range of $\rho^{P}$, is therefore seen to be a necessary condition for separability, called the range criterion. ${ }^{6}$ Since there exist entangled states satisfying the range criterion, the condition is not sufficient. 
Please note that the range criterion demands that there should exist at least one such set of product vectors, not that all product vectors in the ranges should be related by partial conjugation. For example, in $3 \times 3$ dimensions, which is the case discussed here, when a separable state $\rho$ is a convex combination of five randomly chosen pure product states, $\operatorname{Img} \rho$ and $\operatorname{Img} \rho^{P}$ will contain exactly six product vectors each, but the sixth product vector in $\operatorname{Img} \rho^{P}$ is not the partial conjugate of the sixth product vector in $\operatorname{Img} \rho$.

An edge state is defined as a PPT state that breaks the range criterion maximally, in the sense that there exists no product vector in the range of $\rho$ with its partial conjugate in the range of $\rho^{P}$. All edge states are entangled by the range criterion.

It is straightforward to see that every extremal entangled PPT state $\rho$ must be an edge state. In fact, if $w=u \otimes v \in \operatorname{Img} \rho$ and $\tilde{w}=u \otimes v^{*} \in \operatorname{Img} \rho^{P}$, then $\rho$ is not extremal, because $(1-\epsilon) \rho+\epsilon w w^{\dagger}$ is a PPT state for both positive and negative $\epsilon$ in some finite interval. The converse is not true: there exist plenty of edge states that are not extremal. ${ }^{13}$

\subsection{Extremality in $\mathcal{P}$}

We will now describe the extremality test in $\mathcal{P}=\mathcal{D} \cap \mathcal{D}^{P}$, which follows directly from the extremality test in $\mathcal{D}$. We also want to outline how to use perturbations with various restrictions in order to calculate the dimensions of surfaces of states in $\mathcal{P}$ of fixed ranks. Thus, we are interested in perturbations that preserve the ranks $(m, n)$ of $\rho$ and $\rho^{P}$ simultaneously, but do not necessarily preserve the ranges.

As we did for $\rho$, we define $\tilde{P}$ and $\tilde{Q}=I-\tilde{P}$ as the orthogonal projections onto $\operatorname{Img} \rho^{P}$ and $\operatorname{Ker} \rho^{P}$. We then define

$$
\begin{aligned}
\tilde{\mathbf{P}} X & =\left(\tilde{P} X^{P} \tilde{P}\right)^{P}, \\
\tilde{\mathbf{Q}} X & =\left(\tilde{Q} X^{P} \tilde{Q}\right)^{P}, \\
\tilde{\mathbf{R}} X & =(\mathbf{I}-\tilde{\mathbf{P}}-\tilde{\mathbf{Q}}) X .
\end{aligned}
$$

These are again projections on the real Hilbert space $H_{N}$, and like $\mathbf{P}, \mathbf{Q}$ and $\mathbf{R}$, they are symmetric with respect to the natural scalar product on $H_{N}$. We use these projection operators on $H_{N}$ to impose various restrictions on the perturbation matrix $A$ in Eq. (9).

\section{Testing for extremality in $\mathcal{P}$}

Clearly $\rho$ is extremal in $\mathcal{P}$ if and only if $A=\rho$ is the only simultaneous solution of the two equations $\mathbf{P} A=A$ and $\tilde{\mathbf{P}} A=A$. Another way to formulate this condition is that there exists no $\rho^{\prime} \in \mathcal{P}, \rho^{\prime} \neq \rho$ with both $\operatorname{Img} \rho^{\prime}=\operatorname{Img} \rho$ and $\operatorname{Img}$ $\left(\rho^{\prime}\right)^{P}=\operatorname{Img} \rho^{P}$. 
Since $\mathbf{P}$ and $\tilde{\mathbf{P}}$ are projections, the equations $\mathbf{P} A=A$ and $\tilde{\mathbf{P}} A=A$ together are equivalent to the single eigenvalue equation

$$
(\mathbf{P}+\tilde{\mathbf{P}}) A=2 A .
$$

Note that the operator $\mathbf{P}+\tilde{\mathbf{P}}$ is real symmetric and positive and therefore has a complete set of nonnegative real eigenvalues and eigenvectors.

When we diagonalize $\mathbf{P}+\tilde{\mathbf{P}}$, we will always find $A=\rho$ as an eigenvector with eigenvalue 2 . If it is the only solution of Eq. (28), this proves that $\rho$ is extremal in $\mathcal{P}$. If $A$ is a solution not proportional to $\rho$, then we may impose the condition $\operatorname{Tr} A=0$ (replace $A$ by $A-(\operatorname{Tr} A) \rho$ if necessary), and we know that there exists a finite range of both positive and negative values of $\epsilon$ such that $\rho^{\prime}=\rho+\epsilon A \in \mathcal{P}$, hence $\rho$ is not extremal in $\mathcal{P}$.

\section{Perturbations preserving the PPT property and ranks}

The rank and positivity of $\rho$ is preserved by the perturbation $\rho^{\prime}=\rho+\epsilon A$ to first order in $\epsilon$, both for $\epsilon>0$ and $\epsilon<0$, if and only if $\mathbf{Q} A=0$. Similarly, the rank and positivity of $\rho^{P}$ is preserved if and only if $\tilde{\mathbf{Q}} A=0$. These two equations together are equivalent to the single eigenvalue equation

$$
(\mathbf{Q}+\tilde{\mathbf{Q}}) A=0
$$

Again, $\mathbf{Q}+\tilde{\mathbf{Q}}$ is real symmetric and has a complete set of real eigenvalues and eigenvectors. The number of linearly independent solutions for $A$ in Eq. (29) is then the dimension of the surface through $\rho$ of rank $(m, n)$ PPT states.

We may want to perturb in such a way that $\operatorname{Img} \rho^{\prime}=\operatorname{Img} \rho$, but not necessarily $\operatorname{Img}\left(\rho^{\prime}\right)^{P}=\operatorname{Img} \rho^{P}$, that is, we only require $\operatorname{Img}\left(\rho^{\prime}\right)^{P}$ and $\operatorname{Img} \rho^{P}$ to have the same rank. Then, the conditions on $A$ are that $\mathbf{P} A=A$ and $\tilde{\mathbf{Q}} A=0$, or equivalently

$$
(\mathbf{I}-\mathbf{P}+\tilde{\mathbf{Q}}) A=0 .
$$

In this case, the number of linearly independent solutions for $A$ is the dimension of the surface through $\rho$ of rank $(m, n) \mathrm{PPT}$ states with fixed range Img $\rho$.

\subsection{Product transformations}

A product transformation of the form

$$
\rho \mapsto \rho^{\prime}=a V \rho V^{\dagger}, \quad V=V_{A} \otimes V_{B},
$$

where $a>0$ is a normalization factor and $V_{A} \in \mathrm{SL}\left(N_{A}, \mathbb{C}\right), V_{B} \in \operatorname{SL}\left(N_{B}, \mathbb{C}\right)$, preserves positivity, rank, separability and other interesting properties that the density matrix $\rho$ may have. It preserves positivity of the partial transpose because

$$
\left(\rho^{\prime}\right)^{P}=a \tilde{V} \rho^{P} \tilde{V}^{\dagger}, \quad \tilde{V}=V_{A} \otimes V_{B}^{*} .
$$


A transformation of the form of Eq. (31) is also sometimes referred to as a local SL-transformation.

The range and kernel of $\rho$ and $\rho^{P}$ transform in the following ways:

$$
\begin{gathered}
\operatorname{Img} \rho^{\prime}=V \operatorname{Img} \rho, \quad \operatorname{Ker} \rho^{\prime}=\left(V^{\dagger}\right)^{-1} \operatorname{Ker} \rho, \\
\operatorname{Img}\left(\rho^{\prime}\right)^{P}=\tilde{V} \operatorname{Img} \rho^{P}, \quad \operatorname{Ker}\left(\rho^{\prime}\right)^{P}=\left(\tilde{V}^{\dagger}\right)^{-1} \operatorname{Ker} \rho^{P} .
\end{gathered}
$$

We say that two density matrices $\rho$ and $\rho^{\prime}$ related in this way are SL $\otimes$ SL-equivalent. The concept of SL $\otimes$ SL-equivalence is important because it simplifies very much the classification of the low rank PPT states. Since this SL $\otimes$ SL-equivalence is transitive, it generates equivalence classes of matrices.

\section{$\mathrm{SL} \otimes \mathrm{SL}-$ symmetry under partial transposition}

We say that the state $\rho$ is $\mathrm{SL} \otimes \mathrm{SL}$-symmetric if $\rho$ and $\rho^{P}$ are $\mathrm{SL} \otimes \mathrm{SL}$-equivalent, that is, if

$$
\rho^{P}=a V \rho V^{\dagger}, \quad V=V_{A} \otimes V_{B} .
$$

Since SL-transformations of product type $V=V_{A} \otimes V_{B}$ preserve the number of product vectors in a subspace, any transformation $\rho \mapsto \rho^{P}=a V \rho V^{\dagger}$ must transform the set of $n_{\text {img }}$ product vectors in the range of $\rho$ to the set of $\tilde{n}_{\text {img }}$ product vectors in the range of $\rho^{P}$, so for SL $\otimes$ SL-symmetric states, we must have $n_{\text {img }}=\tilde{n}_{\text {img }}$. If the product vectors in $\operatorname{Img} \rho$ are $w_{i}=u_{i} \otimes v_{i}$ with $i=1, \ldots, n_{\text {img }}$ and the product vectors in $\operatorname{Img} \rho^{P}$ are $\tilde{w}_{i}=\tilde{u}_{i} \otimes \tilde{v}_{i}$ for $i=1, \ldots, n_{\text {img }}$, then

$$
V_{A} u_{i}=\tilde{u}_{i}, \quad V_{B} v_{i}=\tilde{v}_{i} .
$$

Since our understanding of the relation between the sets $\left\{w_{i}\right\}$ and $\left\{\tilde{w}_{i}\right\}$ is quite limited for entangled states, it is difficult to say much in general about what makes some states SL $\otimes$ SL-symmetric and others not.

\section{Genuine $\mathbf{S L} \otimes \mathbf{S L}$-symmetry}

We say that a state $\rho$ is genuinely $\mathrm{SL} \otimes \mathrm{SL}$-symmetric if there exists a transformation

$$
\rho^{\prime}=a U \rho U^{\dagger}, \quad U=U_{A} \otimes U_{B},
$$

such that $\left(\rho^{\prime}\right)^{P}=\rho^{\prime}$. The transformation of $\rho$ implies that

$$
\left(\rho^{\prime}\right)^{P}=a \tilde{U} \rho^{P} \tilde{U}^{\dagger}
$$

when we define $\tilde{U}=U_{A} \otimes U_{B}^{*}$. The genuine SL $\otimes$ SL-symmetry implies further that

$$
\tilde{U} \rho^{P} \tilde{U}^{\dagger}=U \rho U^{\dagger}
$$


and hence

$$
\rho^{P}=V \rho V^{\dagger} \quad \text { with } V=\tilde{U}^{-1} U=I \otimes V_{B}
$$

and with $V_{B}=\left(U_{B}^{*}\right)^{-1} U_{B}$. This shows that genuine SL $\otimes$ SL-symmetry implies $\mathrm{SL} \otimes \mathrm{SL}$-symmetry, and Eq. (39) requires that $V$ preserves the trace of $\rho$,

$$
\operatorname{Tr} \rho=\operatorname{Tr} \rho^{P}=\operatorname{Tr}\left(V \rho V^{\dagger}\right)=\operatorname{Tr}\left(\rho V^{\dagger} V\right) .
$$

A sufficient but not necessary condition for this trace preservation is that $V_{B}$ is unitary.

The relation $V_{B}=\left(U_{B}^{*}\right)^{-1} U_{B}$ implies that $V_{B}$ has some special properties. One implication is that $V_{B}^{*}=V_{B}^{-1}$ and hence $\left|\operatorname{det} V_{B}\right|=1$. If we multiply $U_{B}$ by some phase factor $\mathrm{e}^{\mathrm{i} \alpha}$, then $V_{B}$ is multiplied by $\mathrm{e}^{2 \mathrm{i} \alpha}$, and in this way, we may redefine $V_{B}$ such that $\operatorname{det} V_{B}=1$.

We conclude that for the state $\rho$ to be genuinely $\mathrm{SL} \otimes \mathrm{SL}$-symmetric, it must be $\mathrm{SL} \otimes \mathrm{SL}-$ symmetric with a transformation of the form given in Eq. (39). For the $3 \times 3$ system, $V$ would have the form

$$
V=\left(\begin{array}{ccc}
V_{B} & 0 & 0 \\
0 & V_{B} & 0 \\
0 & 0 & V_{B}
\end{array}\right)
$$

with $V_{B} \in \mathrm{SL}(3, \mathbb{C})$ and $V_{B}^{*}=V_{B}^{-1}$.

Since the transformation has the form $V=I \otimes V_{B}$ in the case of genuine $\mathrm{SL} \otimes \mathrm{SL}$-symmetry, by Eq. (35), the product vectors in $\operatorname{Img} \rho$ and $\operatorname{Img} \rho^{P}$ will be related by the transformations $\tilde{u}_{i}=u_{i}$ and $\tilde{v}_{i}=V_{B} v_{i}$. This is a necessary condition for genuine $\mathrm{SL} \otimes \mathrm{SL}$-symmetry which may be tested as soon as we know the product vectors in $\operatorname{Img} \rho$ and $\operatorname{Img} \rho^{P}$.

Assume that for a given PPT state $\rho$, we find that $\rho$ and $\rho^{P}$ are related by a transformation of the form given in Eq. (39). Then, a further problem to be solved is to find a transformation $U=U_{A} \otimes U_{B}$ that demonstrates explicitly the genuine $\mathrm{SL} \otimes \mathrm{SL}$-symmetry of $\rho$. Here $U_{A}$ is completely arbitrary, hence the simplest solution is to take $U_{A}=I$. Next, we have to solve the equation $V_{B}=\left(U_{B}^{*}\right)^{-1} U_{B}$ for $U_{B}$. We find one particular solution by assuming that $U_{B}^{*}=U_{B}^{-1}$. This gives the equation $V_{B}=\left(U_{B}\right)^{2}$, and the solution is the matrix square root,

$$
U_{B}=\sqrt{V_{B}}
$$

Since $V_{B}^{*}=V_{B}^{-1}$, this is consistent with the assumption $U_{B}^{*}=U_{B}^{-1}$.

It should be noted that the matrix square root is in general many valued. A standard solution $U_{B}=X$ is found by the rapidly converging Newton-Raphson 
method: starting with $X=V_{B}$, we iterate the substitution

$$
X \rightarrow X^{\prime}=\frac{1}{2}\left(X+X^{-1} V_{B}\right) .
$$

We find in practice that this solution for $U=I \otimes U_{B}$ always works.

\section{The Rank $(4,4)$ Extremal PPT States Revisited}

The rank $(4,4)$ extremal PPT states in dimension $3 \times 3$ are well understood. ${ }^{10,11,16,17}$ They are all SL $\otimes$ SL-equivalent to states constructed from UPBs, and they all have exactly six product vectors in their kernels. A construction method not using UPBs was discussed in Ref. 17, and as part of that discussion, the structure of a PPT state $\rho$ with at least four product vectors in $\operatorname{Ker} \rho$ was derived.

In the present section, we will review and expand on the discussion given in Ref. 17. This is relevant for our present study of nongeneric rank $(5,5)$ PPT states, and it leads to a new construction of the rank $(4,4)$ extremal PPT states.

Given a PPT state $\rho$ of rank at most five, and four product vectors $z_{j}=x_{j} \otimes y_{j}$ in Ker $\rho$, in some definite but arbitrary order. We assume that any three $x$ vectors and any three $y$ vectors are linearly independent. Then, we may perform a product transformation as in Eq. (31), and subsequent normalizations, so that the vectors take the form

$$
x=y=\left(\begin{array}{llll}
1 & 0 & 0 & 1 \\
0 & 1 & 0 & 1 \\
0 & 0 & 1 & 1
\end{array}\right)
$$

and

$$
z=\left(\begin{array}{llll}
1 & 0 & 0 & 1 \\
0 & 0 & 0 & 1 \\
0 & 0 & 0 & 1 \\
0 & 0 & 0 & 1 \\
0 & 1 & 0 & 1 \\
0 & 0 & 0 & 1 \\
0 & 0 & 0 & 1 \\
0 & 0 & 0 & 1 \\
0 & 0 & 1 & 1
\end{array}\right) .
$$

The transformation is unique. In this four-dimensional subspace, there exist no other product vectors. The real form of the product vectors $z_{j} \in \operatorname{Ker} \rho$ implies that $z_{j} \in \operatorname{Ker} \rho^{P}$.

It is equally easy to see that there exist exactly six product vectors $w_{i}=u_{i} \otimes v_{i}$ in the orthogonal subspace. In fact, in order to have $\left(u_{i} \otimes v_{i}\right) \perp\left(x_{j} \otimes y_{j}\right)$ for all $i=$ $1, \ldots, 6$ and $j=1, \ldots, 4$, we must have for each pair $i, j$ that either $u_{i} \perp x_{j}$ or $v_{i} \perp y_{j}$. 
Table 1. The six possible ways to have a product vector $u_{i} \otimes v_{i}$ orthogonal to all four product vectors $x_{j} \otimes y_{j}$.

\begin{tabular}{ccc}
\hline$u_{i} \otimes v_{i}$ & $u_{i} \perp x_{k}, x_{l}$ & $v_{i} \perp y_{m}, y_{n}$ \\
\hline$i$ & $k, l$ & $m, n$ \\
\hline 1 & 2,3 & 1,4 \\
2 & 1,3 & 2,4 \\
3 & 1,2 & 3,4 \\
4 & 1,4 & 2,3 \\
5 & 2,4 & 1,3 \\
6 & 3,4 & 1,2 \\
\hline
\end{tabular}

Since any three $x$ vectors and any three $y$ vectors are linearly independent, a $u$ vector can be orthogonal to at most two $x$ vectors, and a $v$ vector can be orthogonal to at most two $y$ vectors. This gives the six possibilities for orthogonality listed in Table 1.

The unique solutions are the following:

$$
\begin{aligned}
u=\left(\begin{array}{rrrrrr}
1 & 0 & 0 & 0 & 1 & 1 \\
0 & 1 & 0 & 1 & 0 & -1 \\
0 & 0 & 1 & -1 & -1 & 0
\end{array}\right), \quad v=\left(\begin{array}{rrrrrrrr}
0 & 1 & 1 & 1 & 0 & 0 \\
1 & 0 & -1 & 0 & 1 & 0 \\
-1 & -1 & 0 & 0 & 0 & 1
\end{array}\right), \\
w=\left(\begin{array}{rrrrrr}
0 & 0 & 0 & 0 & 0 & 0 \\
1 & 0 & 0 & 0 & 1 & 0 \\
-1 & 0 & 0 & 0 & 0 & 1 \\
0 & 1 & 0 & 1 & 0 & 0 \\
0 & 0 & 0 & 0 & 0 & 0 \\
0 & -1 & 0 & 0 & 0 & -1 \\
0 & 0 & 1 & -1 & 0 & 0 \\
0 & 0 & -1 & 0 & -1 & 0 \\
0 & 0 & 0 & 0 & 0 & 0
\end{array}\right) .
\end{aligned}
$$

The five-dimensional subspace $\operatorname{Span} w$ given by $(47)$ defines a face $\mathcal{F} \subset \mathcal{D}$ of dimension $5^{2}-1=24$. Recall that

$$
\rho z_{j}=\rho^{P} z_{j}=0, \quad j=1,2,3,4 .
$$

In terms of the face $\mathcal{F}$, Eq. (48) means that $\rho \in \mathcal{F}$ and $\rho^{P} \in \mathcal{F}$, or equivalently, $\rho \in \mathcal{F} \cap \mathcal{F}^{P}$. Equation (48) restricts $\rho$ to have the following form, with real 
coefficients $c_{i}$ :

$$
\rho=\frac{1}{2}\left(\begin{array}{ccccccccc}
0 & 0 & 0 & 0 & 0 & 0 & 0 & 0 & 0 \\
0 & c_{1}+c_{5} & -c_{1} & 0 & 0 & 0 & 0 & -c_{5} & 0 \\
0 & -c_{1} & c_{1}+c_{6} & 0 & 0 & -c_{6} & 0 & 0 & 0 \\
0 & 0 & 0 & c_{2}+c_{4} & 0 & -c_{2} & -c_{4} & 0 & 0 \\
0 & 0 & 0 & 0 & 0 & 0 & 0 & 0 & 0 \\
0 & 0 & -c_{6} & -c_{2} & 0 & c_{2}+c_{6} & 0 & 0 & 0 \\
0 & 0 & 0 & -c_{4} & 0 & 0 & c_{3}+c_{4} & -c_{3} & 0 \\
0 & -c_{5} & 0 & 0 & 0 & 0 & -c_{3} & c_{3}+c_{5} & 0 \\
0 & 0 & 0 & 0 & 0 & 0 & 0 & 0 & 0
\end{array}\right) .
$$

This form implies that $\rho^{P}=\rho$. Since $\mathcal{F}$ is a face on $\mathcal{D}$, we see that $\mathcal{F}^{P}$ is a face on $\mathcal{D}^{P}$, and the intersection $\mathcal{G}=\mathcal{F} \cap \mathcal{F}^{P}$ is a face on $\mathcal{P}=\mathcal{D} \cap \mathcal{D}^{P}$. Equation (49) with the normalization condition

$$
\operatorname{Tr} \rho=\sum_{i=1}^{6} c_{i}=1
$$

shows that the face $\mathcal{G}$ has dimension five.

The state $\rho$ defined in Eq. (49) is a linear combination, but not necessarily a convex combination, of the six pure product states,

$$
\rho=\frac{1}{2} \sum_{i=1}^{6} c_{i} w_{i} w_{i}^{\dagger} .
$$

Since the matrices $w_{i} w_{i}^{\dagger}$ are linearly independent, and there are no other pure product states in $\operatorname{Img} \rho$, we see that $\rho$ is separable if and only if all the coefficients $c_{i}$ are nonnegative. However, we will now see that it is possible for $\rho$ to be an entangled PPT state even if one of the coefficients is negative.

An eigenvalue of $\rho$, and of $\rho^{P}=\rho$, is a root of the characteristic polynomial

$$
\operatorname{det}(\rho-\lambda I)=-\lambda^{4}\left(\lambda^{5}-d_{4} \lambda^{4}+d_{3} \lambda^{3}-d_{2} \lambda^{2}+d_{1} \lambda-d_{0}\right)
$$

with

$$
d_{0}=\frac{3}{16} \sum_{i=1}^{6} \prod_{j \neq i} c_{j}=\frac{3}{16}\left(\prod_{j=1}^{6} c_{j}\right) \sum_{i=1}^{6} \frac{1}{c_{i}} .
$$

If we start with $c_{i}>0$ for $i=1, \ldots, 6$, then $\rho$ is a rank $(5,5)$ separable state. If we next change the coefficients continuously, $\rho$ will continue to have five positive eigenvalues until we get $d_{0}=0$. Hence, the equation $d_{0}=0$ defines the boundary of the set of density matrices and also of the set of PPT states since $\rho^{P}=\rho$.

We know that the boundary is not reached before at least one coefficient $c_{i}$ becomes zero or negative. If two coefficients become zero simultaneously, then $d_{0}=0$ 
and we have reached a boundary state which is separable. To get negative coefficients while $\rho$ is a rank $(5,5)$ PPT state, we have to make one coefficient negative before the others. Let us say, for example, that $c_{1}<0$, and that we want to make also $c_{2}$ negative, while $c_{i}>0$ for $i=3,4,5,6$. Then, we first have to make $c_{2}=0$, in which case $d_{0}=3 c_{1} c_{3} c_{4} c_{5} c_{6} / 16<0$ and we have already crossed the boundary $d_{0}=0$.

In conclusion, the entangled boundary states have $c_{i} \neq 0$ for $i=1, \ldots, 6$, and they have one negative and five positive coefficients $c_{i}$ satisfying the equation

$$
\sum_{i=1}^{6} \frac{1}{c_{i}}=0 .
$$

Thus, the boundary $d_{0}=0$ consists of two types of states:

(1) Separable states that are convex combinations of up to four of the pure product states $w_{i} w_{i}^{\dagger}$.

(2) Rank $(4,4)$ entangled PPT states that are linear combinations of all the six pure product states $w_{i} w_{i}^{\dagger}$ with exactly one negative coefficient.

It is well known that rank $(4,4)$ entangled PPT states are extremal.

Figure 1 shows an example of a two-dimensional section through the fivedimensional face of $\mathcal{P}$ defined by Eqs. (50) and (51). The section through $\mathcal{S}$ is the hexagon with corners $A$ to $F$. In Table 2, we have listed the coefficients $c_{i}$, multiplied

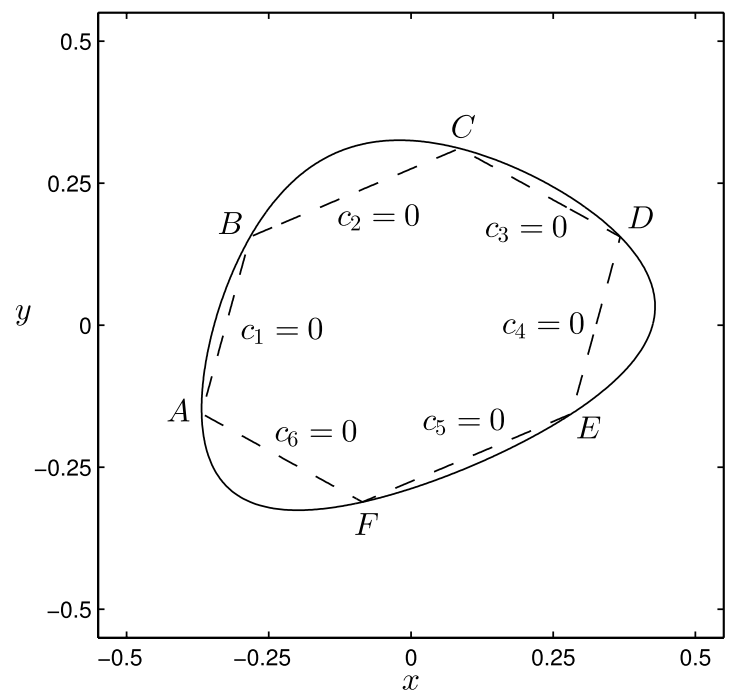

Fig. 1. A two-dimensional section through the five-dimensional face on the set of PPT states consisting of normalized density matrices of the form in Eq. (49). The outer curve is the common boundary of $\mathcal{D}$ and $\mathcal{P}$, it consists of rank $(4,4)$ PPT states. The hexagon (dashed) is the boundary of the simplex of separable states, where one of the coefficients $c_{i}$ is zero. The region between the two curves consists of entangled PPT states of rank $(5,5)$ with one coefficient negative. The coefficients $c_{i}$ defining the separable boundary states $A$ to $F$ are given in Table 2 . 
Table 2. The coefficients $c_{i}$, multiplied by 12 , for the states $A-F$ in Fig. 1.

\begin{tabular}{lllllll}
\hline$i$ & $A$ & $B$ & $C$ & $D$ & $E$ & $F$ \\
\hline 1 & 0 & 0 & 3 & 6 & 6 & 3 \\
2 & 1 & 0 & 0 & 1 & 2 & 2 \\
3 & 6 & 3 & 0 & 0 & 3 & 6 \\
4 & 2 & 2 & 1 & 0 & 0 & 1 \\
5 & 3 & 6 & 6 & 3 & 0 & 0 \\
6 & 0 & 1 & 2 & 2 & 1 & 0 \\
\hline
\end{tabular}

by 12 , that define the states $A$ to $F$ by Eq. (51). The hexagon is reflection symmetric about two axes.

\section{The most general rank $(4,4)$ entangled PPT states}

Consider a general rank $(4,4)$ entangled PPT state $\rho$ in $3 \times 3$ dimensions. It is known that any such state is extremal and has exactly six product vectors in its kernel. We can now see that it is SL $\otimes$ SL-equivalent, in no less than 360 different ways, to such states on the boundary of the five-dimensional face of $\mathcal{P}$ that we have described here. The 360 transformations are found in the following way.

Pick any four of the six product vectors in Ker $\rho$, which can be done in 15 different ways. Order them next in one of the 24 possible ways. Altogether, there are $24 \times 15=$ 360 possibilities. There is then a unique product transformation that transforms the four product vectors to the form given in Eq. (45). We know that it must transform the state $\rho$ into one of the rank $(4,4)$ states described by the Eqs. (50), (51) and (54), since these are the only rank $(4,4)$ entangled PPT states of this form.

\section{Rank $(5,5)$ PPT States in $3 \times 3$ Dimensions}

Our main purpose with the present study is to try to understand the rank $(5,5)$ entangled PPT states in $3 \times 3$ dimensions. In particular, we would like to understand better the relation between $\operatorname{Img} \rho$ and $\operatorname{Img} \rho^{P}$ when $\rho$ is a rank $(5,5)$ PPT state. A natural question is whether $\rho$ is $\mathrm{SL} \otimes \mathrm{SL}-$ symmetric, as defined in Eq. (34), so that $\operatorname{Img} \rho^{P}=V \operatorname{Img} \rho$ with $V=V_{A} \otimes V_{B}$.

The product vectors in $\operatorname{Img} \rho$ and $\operatorname{Img} \rho^{P}$ are very useful for answering the question of SL $\otimes$ SL-symmetry, especially when both these spaces have dimension five. By Eq. (16), the number of constraints to be satisfied by a product vector is $K=4$, thus $K+1=5$ is precisely the critical dimension at which every subspace contains one or more product vectors, and a generic subspace contains a finite number of product vectors, exactly six in this case. From these product vectors, one may construct invariants that may be used to test whether the two spaces are related by some product transformation $V=V_{A} \otimes V_{B}$, which is a necessary condition for the $\mathrm{SL} \otimes \mathrm{SL}-$ symmetry of the state $\rho$. 


\subsection{Generic five-dimensional subspaces}

Any set of five product vectors $w_{i}=u_{i} \otimes v_{i}$ in a generic five-dimensional subspace may be transformed by an SL $\otimes$ SL-transformation, followed by suitable normalizations, to the standard form ${ }^{17}$

$$
u=\left(\begin{array}{lllll}
1 & 0 & 0 & 1 & 1 \\
0 & 1 & 0 & 1 & p \\
0 & 0 & 1 & 1 & q
\end{array}\right), \quad v=\left(\begin{array}{ccccc}
1 & 0 & 0 & 1 & 1 \\
0 & 1 & 0 & 1 & r \\
0 & 0 & 1 & 1 & s
\end{array}\right)
$$

with $p, q, r, s$ as real or complex parameters. By generic, we here mean that any three vectors in $u$ and in $v$ are linearly independent. There will also be a sixth product vector $w_{6}=u_{6} \otimes v_{6}$ which is a linear combination of the above five. The parameters $p, q, r, s$ are determined by the following ratios of determinants,

$$
\begin{gathered}
s_{1}=-\frac{\operatorname{det}\left(u_{1} u_{2} u_{4}\right) \operatorname{det}\left(u_{1} u_{3} u_{5}\right)}{\operatorname{det}\left(u_{1} u_{2} u_{5}\right) \operatorname{det}\left(u_{1} u_{3} u_{4}\right)}=-\frac{p}{q}, \\
s_{2}=-\frac{\operatorname{det}\left(u_{1} u_{2} u_{3}\right) \operatorname{det}\left(u_{2} u_{4} u_{5}\right)}{\operatorname{det}\left(u_{1} u_{2} u_{4}\right) \operatorname{det}\left(u_{2} u_{3} u_{5}\right)}=q-1, \\
s_{3}=\frac{\operatorname{det}\left(v_{1} v_{2} v_{3}\right) \operatorname{det}\left(v_{1} v_{4} v_{5}\right)}{\operatorname{det}\left(v_{1} v_{2} v_{5}\right) \operatorname{det}\left(v_{1} v_{3} v_{4}\right)}=\frac{r-s}{s}, \\
s_{4}=\frac{\operatorname{det}\left(v_{1} v_{3} v_{5}\right) \operatorname{det}\left(v_{2} v_{3} v_{4}\right)}{\operatorname{det}\left(v_{1} v_{2} v_{3}\right) \operatorname{det}\left(v_{3} v_{4} v_{5}\right)}=\frac{r}{1-r} .
\end{gathered}
$$

All the parameters $s_{i}$ are invariant under SL $\otimes$ SL-transformations. Hence, for given vectors $u_{i}$ and $v_{i}$ not on standard form, these formulas may be used to calculate the values of the parameters $p, q, r, s$ without actually performing the transformation to standard form.

Though only $u_{1}, \ldots, u_{5}$ and $v_{1}, \ldots, v_{5}$ occur in Eqs. (56) and (57), since the numbering is arbitrary, all six product vectors must be taken into consideration when calculating the invariants. Different permutations of the six product vectors will in general give different values for the invariants.

The standard form in Eq. (55), or equivalently the invariants $s_{i}$ defined in Eqs. (56) and (57), can be used to check whether $\rho$ and $\rho^{P}$ are SL $\otimes$ SL-equivalent. We must find the six product vectors in $\operatorname{Img} \rho$ and $\operatorname{Img} \rho^{P}$ in some order. Then, we either transform these to the standard form in Eq. (55), or calculate directly the invariants $s_{i}$ for both subspaces. For the comparison, we should try all the $6 !=720$ permutations of the six product vectors in one of the two subspaces. If the invariants so calculated are identical for a given permutation, then $\operatorname{Img} \rho$ and $\operatorname{Img} \rho^{P}$ can be transformed by a unique SL $\otimes$ SL-transformation to the standard form in Eq. (55) with the same values of $p, q, r, s$. The transformations of both spaces to a common standard form then define a unique transformation $V=V_{A} \otimes V_{B}$ from $\operatorname{Img} \rho$ to $\operatorname{Img} \rho^{P}$. It is then easy to check whether $\rho^{P}=a V \rho_{1} V^{\dagger}$ for some $a>0$. 
Note that different permutations may give identical sets of invariants, but different $V$ that transform between the two spaces. We may have to try all of these transformations in order to find one that transforms $\rho$ into $\rho^{P}$.

Also note that the partial transpose of $\rho$ with respect to subsystem $A$ is $\left(\rho^{P}\right)^{*}$. If $\rho$ is $\mathrm{SL} \otimes \mathrm{SL}$-symmetric under this partial transposition, then it means that the invariants of $\operatorname{Img} \rho^{P}$ will be the complex conjugates of the invariants of $\operatorname{Img} \rho$. In general, this type of symmetry is just as likely to occur.

\section{Separable rank $(5,5)$ states}

In a generic five-dimensional subspace of $\mathbb{C}^{9}$ containing six normalized product vectors $w_{i}=u_{i} \otimes v_{i}$, we may construct a five-dimensional set of separable states as convex combinations

$$
\rho=\sum_{i=1}^{6} c_{i} w_{i} w_{i}^{\dagger}
$$

with $c_{i} \geq 0$ and $\sum_{i} c_{i}=1$. Hence, all the separable states in the subspace are contained in a simplex with the six pure product states as vertices. The partial transpose of $\rho$ is

$$
\rho^{P}=\sum_{i=1}^{6} c_{i} \tilde{w}_{i} \tilde{w}_{i}^{\dagger},
$$

where $\tilde{w}_{i}=u_{i} \otimes v_{i}^{*}$ is the partial conjugate of $w_{i}$. The six partially conjugated product vectors will be linearly independent in the generic case, hence the separable states in the interior of the simplex will have rank $(5,6)$.

On the boundary where one coefficient $c_{i}$ vanishes, $\rho$ will be a rank $(5,5) \mathrm{PPT}$ state. In this case, five of the product vectors $\operatorname{in} \operatorname{Img} \rho$ and in $\operatorname{Img} \rho^{P}$ are partial conjugates of each other, whereas the sixth product vectors in the two spaces are related in a more complicated way, unless all the vectors $v_{i}$ are real.

\section{The surface of generic rank $(5,5)$ PPT states}

It is known that a generic four-dimensional subspace is not the range of any entangled rank $(4,4)$ PPT state. ${ }^{10}$ It appears however in our numerical investigations that every generic five-dimensional subspace is the common range of extremal and hence entangled rank $(5,5)$ PPT states that together form an eight-dimensional surface. ${ }^{17}$ It is an interesting numerical observation that the dimension of the surface of rank $(5,5) \mathrm{PPT}$ states in a generic five-dimensional subspace is higher than the dimension of the simplex of separable states.

In order to compute this surface numerically, we consider the perturbation

$$
\rho^{\prime}=\rho+\epsilon A
$$

with $\operatorname{Tr} A=0$ and $A$ satisfying Eq. (30). 
We find eight linearly independent solutions for $A$ in addition to the trivial solution $A=\rho$, meaning that the surface has dimension eight.

Similarly, if we want to find the dimension of the total set of rank $(5,5) \mathrm{PPT}$ states, we find that Eq. (29) has 48 linearly independent nontrivial solutions. The dimensions 8 and 48 are consistent with the fact that the set of five-dimensional subspaces has dimension 40, see Ref. 17.

\subsection{Nongeneric five-dimensional subspaces}

By definition, for a generic set of vectors in $\mathbb{C}^{3}$, any subset of three vectors will be linearly independent. For a generic $\operatorname{rank}(5,5) \mathrm{PPT}$ state $\rho$, the range Img $\rho$ contains six product vectors $w_{i}=u_{i} \otimes v_{i}$. A nonzero vector $x \in \mathbb{C}^{3}$ can at most be orthogonal to two vectors $u_{i}$, and a nonzero $y$ can at most be orthogonal to two $v_{i}$, hence $z=x \otimes y$ can at most be orthogonal to four $w_{i}$. Since $\operatorname{Ker} \rho=(\operatorname{Img} \rho)^{\perp}$, it is clear that in the generic case, it is not possible to have a product vector in $\operatorname{Ker} \rho$, as described in Sec. 3.3. Thus, generic states must have $n_{\mathrm{ker}}=0$.

In order to construct pairs of orthogonal subspaces $\mathcal{U} \subset \mathbb{C}^{9}$ and $\mathcal{V}=\mathcal{U}^{\perp}$ with $|\mathcal{U}|=5$ and $|\mathcal{V}|=4$, such that $\mathcal{V}$ contains one or more product vectors, we must alter the generic linear dependencies of the $u_{i}$ and $v_{i}$ vectors. Instead of the generic condition that any three vectors $u_{i}$, and any three $v_{i}$, must be linearly independent and span $\mathbb{C}^{3}$, we introduce the condition that any four vectors must span $\mathbb{C}^{3}$. Then, it is possible to have one or more product vectors $z_{j}=x_{j} \otimes y_{j}$ with each $x_{j}$ orthogonal to $u_{a}, u_{b}, u_{c}$ and $y_{j}$ orthogonal to $v_{d}, v_{e}, v_{f}$, where $a, b, \ldots, f$ is some permutation of $1,2, \ldots, 6$.

In the above sense, it is the subspaces that are interesting, and to a lesser degree the states themselves. A general characterization of two orthogonal subspaces $\mathcal{U}$ and $\mathcal{V}$ with regard to the number of product vectors they contain is then $\left\{n_{u} ; n_{v}\right\}$.

Since $z_{j}=x_{j} \otimes y_{j} \in \operatorname{Ker} \rho$ if and only if $\tilde{z}_{j}=x_{j} \otimes y_{j}^{*} \in \operatorname{Ker} \rho^{P}$, it is clear that the kernels of $\rho$ and $\rho^{P}$ are related when they contain product vectors. In particular, if $y_{j}$ is real, then $z_{j}=\tilde{z}_{j} \in \operatorname{Ker} \rho^{P}$. As long as $n_{\text {ker }} \leq 4$, which is always the case in the examples we have constructed here, we can always choose a standard form where all the vectors $x_{j}$ and $y_{j}$ are real.

\section{A Summary of Numerical Results}

We summarize here the main results of our numerical investigations. For ease of reference, we number the cases from I to VII.

\subsection{Generic states: Case I}

The generic rank $(5,5)$ PPT state is an extremal and hence entangled $\{6,6 ; 0\}$ state. By definition, a generic state is found in a completely random search for rank $(5,5)$ PPT states. The number of generic rank $(5,5)$ PPT entangled states we have 
generated are in the thousands. None of them are SL $\otimes$ SL-symmetric, and since they are extremal, they are also edge states. The partial transpose $\rho^{P}$ of a generic rank $(5,5) \mathrm{PPT}$ state $\rho$ is again a generic rank $(5,5)$ PPT state.

Unfortunately, we have made little progress in understanding these states. We have no general understanding of the relation between the five-dimensional subspaces $\operatorname{Img} \rho$ and $\operatorname{Img} \rho^{P}$, which both contain six product vectors. Thus, we have found no basis for a more detailed classification, in terms of canonical forms allowing us to use analytical methods in their construction.

\section{2. $\mathrm{SL} \otimes \mathrm{SL}-$ symmetric states: Case Ia}

The complete set of PPT states of rank $(5,5)$ has dimension 48 , and the set of $\mathrm{SL}(3, \mathbb{C}) \otimes \mathrm{SL}(3, \mathbb{C})$-transformations has dimension $16+16=32$, so the set of equivalence classes of rank $(5,5)$ states with respect to $\mathrm{SL} \otimes \mathrm{SL}$-transformations will have dimension 16, see Ref. 17. Hence, in a completely random search for PPT states of rank $(5,5)$, we will never find two states belonging to the same equivalence class. We also find that such a state $\rho$, which we call generic, does never belong to the same $\mathrm{SL} \otimes \mathrm{SL}-$ equivalence class as its partial transpose $\rho^{P}$.

This shows that SL $\otimes$ SL-symmetric states can be found numerically only by conducting restricted searches. We will describe now two different search methods. For some reason that we can only guess, the second method produces states of a more special kind than the first method.

\section{Method I}

An easy general procedure for constructing numerically PPT states of specified rank that are genuinely SL $\otimes$ SL-symmetric is to construct positive matrices that are symmetric under partial transposition and then subject them to random $\mathrm{SL} \otimes \mathrm{SL}-$ transformations.

\section{Method II}

Method I leaves an open question whether there exist PPT states that are $\mathrm{SL} \otimes \mathrm{SL}$-symmetric but not genuinely $\mathrm{SL} \otimes \mathrm{SL}$-symmetric. In order to answer this question, we produced in a random search more than a hundred SL $\otimes$ SL-symmetric PPT states of rank $(5,5)$ together with their associated SL $\otimes$ SL-transformations. We find that 50 of these are $\{6,6 ; 0\}$ states like the generic rank $(5,5)$ states and we will now describe these. Our search was rather special in the sense that we looked for transformations of the form (34) with $a=1$, i.e. transformations that are trace preserving. This choice allowed for the possibility that the states found might be genuinely $\mathrm{SL} \otimes \mathrm{SL}-$ symmetric.

Out of the 50 states, about half are in fact genuinely SL $\otimes$ SL-symmetric, as defined by Eq. (36). To test for genuine SL $\otimes$ SL-symmetry of a state $\rho$, we compare 
the SL $\otimes \mathrm{SL}$ transformation $V$ from $\rho$ to $\rho^{P}$, found during the search, to the special form given in Eq. (41). If it is of the wanted form, we construct the transformation $U=I \otimes U_{B}$ from (42). If it is not of the wanted form, we have to produce more $\mathrm{SL} \otimes \mathrm{SL}$ transformations, until we either find one of the wanted forms or decide that probably none exists.

We consider two transformation matrices to be identical if they differ only by a phase factor. For the SL $\otimes$ SL-symmetric states that are not genuinely $\mathrm{SL} \otimes \mathrm{SL}-$ symmetric, we find only one $\mathrm{SL} \otimes \mathrm{SL}$ transformation from $\rho$ to $\rho^{P}$. By definition, this is never of the form given in Eq. (41). For the states of genuine $\mathrm{SL} \otimes \mathrm{SL}-$ symmetry found by method II, the number of SL $\otimes$ SL transformations we find is always three, and out of these, there is only one that has the form given in Eq. (41).

A special class of states proposed by Chruściński and Kossakowski has what they call the circulant form ${ }^{18}$

$$
\hat{\rho}=\left(\begin{array}{ccc|ccc|ccc}
a_{11} & \cdot & \cdot & \cdot & a_{12} & \cdot & \cdot & \cdot & a_{13} \\
\cdot & b_{11} & \cdot & \cdot & \cdot & b_{12} & b_{13} & \cdot & \cdot \\
\cdot & \cdot & c_{11} & c_{12} & \cdot & \cdot & \cdot & c_{13} & \cdot \\
\hline \cdot & \cdot & c_{21} & c_{22} & \cdot & \cdot & \cdot & c_{23} & \cdot \\
a_{21} & \cdot & \cdot & \cdot & a_{22} & \cdot & \cdot & \cdot & a_{23} \\
\cdot & b_{21} & \cdot & \cdot & \cdot & b_{22} & b_{23} & \cdot & \cdot \\
\hline \cdot & b_{23} & \cdot & \cdot & \cdot & b_{32} & b_{33} & \cdot & \cdot \\
\cdot & \cdot & c_{31} & c_{32} & \cdot & \cdot & \cdot & c_{33} & \cdot \\
a_{31} & \cdot & \cdot & \cdot & a_{32} & \cdot & \cdot & \cdot & a_{33}
\end{array}\right) .
$$

As long as the $3 \times 3$ matrices $A=\left[a_{i j}\right], B=\left[b_{i j}\right]$ and $C=\left[c_{i j}\right]$ are positive, then so is $\hat{\rho}$, since the eigenvalues of $\hat{\rho}$ are the eigenvalues of the submatrices $A, B$ and $C$. There are similar additional constraints on the elements $a_{i j}, b_{i j}$ and $c_{i j}$ in order for $\hat{\rho}$ to be a PPT state.

Interestingly, all the genuinely SL $\otimes$ SL-symmetric PPT states of rank $(5,5)$ which we have produced by method II can be transformed by product transformations to the form (61). This property is more surprising, since none of the states produced by method I has the same property. We will now describe how to find the transformations.

As already mentioned, for such a state $\rho$, we always find three different product transformations $V_{1}, V_{2}$ and $V_{3}$ such that

$$
V_{1} \rho V_{1}^{\dagger}=V_{2} \rho V_{2}^{\dagger}=V_{3} \rho V_{3}^{\dagger}=\rho^{P} .
$$

Exactly one of them is of the form (41). There are six transformations, not necessarily different, of the form

$$
S=S_{A} \otimes S_{B}=V_{j}^{-1} V_{k}, \quad 1 \leq j, k \leq 3, \quad j \neq k,
$$


transforming from Img $\rho$ back to $\operatorname{Img} \rho$. It does not matter which of the six transformations we choose. We find that with suitable normalization, the equation $S^{3}=I$ is satisfied, and we have the following eigenvalue decompositions:

$$
S_{A}=\sum_{i=1}^{3} \lambda_{i} g_{i} g_{i}^{\dagger}, \quad S_{B}=\sum_{i=1}^{3} \mu_{i} h_{i} h_{i}^{\dagger},
$$

where the two sets of three eigenvalues $\left\{\lambda_{i}\right\}$ and $\left\{\mu_{i}\right\}$ are both $\left\{1, \omega, \omega^{2}\right\}$ in arbitrary order with $\omega=\mathrm{e}^{2 \pi \mathrm{i} / 3}$. The eigenvectors $g_{i}$ are orthonormal like the eigenvectors $h_{i}$. The transformation $T=T_{A} \otimes T_{B}$ with

$$
T_{A}^{-1}=T_{A}^{\dagger}=\left[g_{1}, g_{2}, g_{3}\right], \quad T_{B}^{-1}=T_{B}^{\dagger}=\left[h_{1}, h_{2}, h_{3}\right]
$$

is such that either $T \rho T^{\dagger}=\hat{\rho}$ or $T \rho T^{\dagger}=\hat{\rho}^{P}$ depending on the permutation of the eigenvectors of $S_{A}$ and $S_{B}$.

The fact that the genuinely $\mathrm{SL} \otimes \mathrm{SL}$-symmetric $\mathrm{PPT}$ states of $\operatorname{rank}(5,5)$ produced by method II are of a more special type than those produced by method I, in that they can be transformed to the circulant form (61), needs an explanation. We guess that it is due to the existence of three product transformations from $\rho$ to $\rho^{P}$ rather than one. Presumably, it is easier to find a transformation when three solutions exist. Hence, we introduce a bias when we search simultaneously for a state $\rho$ of rank five and a transformation $V=V_{A} \otimes V_{B}$ such that $V \rho V^{\dagger}=\rho^{P}$.

\subsection{Nongeneric states: Cases II-VII}

Our searches for SL $\otimes$ SL-symmetric states have also produced a small number of states that have the nongeneric property, in addition to being SL $\otimes$ SL-symmetric, that they have product vectors in their kernels. In particular, the very special subspace of type $\{2 ; 1\}$ discussed in Sec. 8 is identified from four SL $\otimes$ SL-symmetric states of type $\{2,2 ; 1\}$ found in these random searches.

In Sec. 7, we discuss standard forms of states that are generically not $\mathrm{SL} \otimes \mathrm{SL}$-symmetric, but have the properties that $n_{\mathrm{img}}=n_{u}=6$ and $n_{\mathrm{ker}}=n_{v}>0$. We have generated numerically and studied nongeneric states having from one to four product vectors in the kernel, as discussed in Sec. 5.2. We define standard forms for nongeneric orthogonal subspaces $\mathcal{U}$ and $\mathcal{V}$ of dimensions five and four, respectively, with various numbers $\left\{n_{u} ; n_{v}\right\}$ of product vectors. We take the product vectors in $\mathcal{V}$ to have the standard form given in Eq. (45), using only the first $n_{v}$ vectors. We have then produced a large number of PPT states $\rho$ of $\operatorname{rank}(5,5)$ with $\operatorname{Img} \rho=\mathcal{U}$ and $\operatorname{Ker} \rho=\mathcal{V}$. We have studied the following nongeneric cases.

Case II: $n_{\mathrm{ker}}=1$. Then Ker $\rho$ contains $z_{1}$ from Eq. (45), and Img $\rho$ is defined by Eq. (83). The rank $(5,5)$ PPT states found are all extremal and of type $\{6,6 ; 1\}$.

Case III: $n_{\text {ker }}=1$. This is the special $\{2 ; 1\}$ subspace presented in Sec. 8 , producing both extremal and nonextremal entangled rank $(5,5)$ PPT states. The extremal states are of type $\{2,6 ; 1\}$. Some of the nonextremal states are of type 
$\{2,6 ; 1\}$, others are symmetric under partial transposition and therefore of type $\{2,2 ; 1\}$.

Case IV: $n_{\text {ker }}=2$. Then Ker $\rho$ in the standard form contains $z_{1}, z_{2}$ and $\operatorname{Img} \rho$ is defined by Eq. (75). The rank $(5,5)$ PPT states found are all extremal and of type $\{6,6 ; 2\}$.

Case V: $n_{\text {ker }}=3$, where Ker $\rho$ contains $z_{1}, z_{2}, z_{3}$ and Img $\rho$ is defined by Eq. (67). The rank $(5,5)$ PPT states found are all nonextremal and of type $\{6,6 ; 3\}$.

Case VI: $n_{\text {ker }}=4$. This is the case discussed in Sec. 4 , with Img $\rho$ defined by Eq. (46). It gives a new way of constructing the extremal rank $(4,4)$ PPT states.

Case VII: $n_{\text {ker }}=2$. Ker $\rho$ contains $z_{1}, z_{2}$ from Eq. (45), whereas Img $\rho$ is defined by Eq. (78) and contains an infinite number of product vectors. The resulting $\{\infty, \infty ; 2\}$ states include both extremal and nonextremal states and also some interesting rank $(4,5)$ PPT states. We know that the latter do not exist in generic subspaces. ${ }^{17}$

\section{Table}

Using the projection operators and extremality tests outlined earlier, we can calculate the dimensions of the surfaces defined by Eqs. (28)-(30) for the various types of states. We use the abbreviation $\mathbf{P}+\tilde{\mathbf{P}}$ for the operation that preserves the range of both $\rho$ and $\rho^{P}, \mathbf{Q}+\tilde{\mathbf{Q}}$ for the preservation of the rank of both $\rho$ and $\rho^{P}$, and finally $\mathbf{P}+\tilde{\mathbf{Q}}$ and $\mathbf{Q}+\tilde{\mathbf{P}}$ for the two other projections. The dimension given by $A=\rho$ has been subtracted. Note that for many of the states, the dimension defined by $\mathbf{P}+\tilde{\mathbf{P}}$ is zero, proving that the state is extremal and therefore entangled.

Presented in Table 3 are data for the various random rank $(5,5)$ PPT states we have produced. They were produced in large numbers with the exception of the $\{2,6 ; 1\}$ extremal states, where a total of 15 states were produced. As we would

Table 3. Data for the random rank $(5,5)$ PPT states we have produced based on the standard forms presented in Sec. 7 and the special $\{2 ; 1\}$ subspace in Sec. 8. Case VIIa are PPT states of rank $(4,5)$. For the dimensions of the various surfaces, the trivial solution $A=\rho$ has been subtracted, so the states with the entry zero for $\mathbf{P}+\tilde{\mathbf{P}}$ are extremal.

\begin{tabular}{lccccccc}
\hline Case & $\left\{n_{\text {img }}, \tilde{n}_{\text {img }} ; n_{\text {ker }}\right\}$ & $\mathbf{Q}+\tilde{\mathbf{Q}}$ & $\mathbf{P}+\tilde{\mathbf{Q}}$ & $\mathbf{Q}+\tilde{\mathbf{P}}$ & $\mathbf{P}+\tilde{\mathbf{P}}$ & Edge & Range \\
\hline I, Ia & $\{6,6 ; 0\}$ & 48 & 8 & 8 & 0 & Yes & No \\
II & $\{6,6 ; 1\}$ & 49 & 9 & 9 & 0 & Yes & No \\
III & $\{2,6 ; 1\}$ & 49 & 9 & 9 & 0 & Yes & No \\
IV & $\{6,6 ; 2\}$ & 50 & 10 & 10 & 0 & Yes & No \\
V & $\{6,6 ; 3\}$ & 51 & 11 & 11 & 3 & No & No \\
VI & $\{6,6 ; 4\}$ & 52 & 12 & 12 & 5 & No & Yes \\
VII & $\{\infty, \infty ; 2\}$ & 50 & 12 & 12 & 0 & Yes & No \\
& $\{\infty, \infty ; 2\}$ & 50 & 11 & 11 & 6 & No & No \\
VIIa & $\{\infty, \infty ; 2\}$ & 50 & 15 & 15 & 9 & No & No \\
\hline
\end{tabular}


expect, none of the states found were $\mathrm{SL} \otimes \mathrm{SL}$-symmetric, except in case Ia where the search was restricted to $\mathrm{SL} \otimes \mathrm{SL}-$ symmetric states.

In addition to the dimensions defined above, we also indicate whether the states are edge states and whether they satisfy the range criterion, as defined in Sec. 3.4.

\section{Nongeneric Rank $(5,5)$ PPT States}

We present here several standard forms for nongeneric orthogonal subspaces $\mathcal{U}=$ $\operatorname{Img} \rho$ and $\mathcal{V}=\operatorname{Ker} \rho$ of dimensions five and four. The set of parameters $a_{i}, b_{i}, c_{i}, d_{i}$, $e_{i}, f_{i}$ is usually assumed to be chosen in a generic or completely random manner. One may however also investigate certain nongeneric choices for these coefficients.

These standard forms can be used to construct nongeneric rank $(5,5) \mathrm{PPT}$ states with a range defined by the given standard form and with $n_{\text {ker }}>0$. Some of these constructions give states that generically are extremal in $\mathcal{P}$ and therefore entangled, while some return generically only nonextremal states.

\section{Case V: $\boldsymbol{n}_{\mathrm{ker}}=\mathbf{3}$}

Given three product vectors in the kernel of $\rho$, we can make a product transformation so that we get for these $z_{i}=x_{i} \otimes y_{i}$ for $i=1,2,3$ from Eq. (45). We choose the orthogonality relations

$$
\begin{array}{llll}
x_{1} \perp u_{i} & i=2,3,6, & y_{1} \perp v_{i} & i=1,4,5, \\
x_{2} \perp u_{i} & i=1,3,5, & y_{2} \perp v_{i} & i=2,4,6, \\
x_{3} \perp u_{i} & i=1,2,4, & y_{3} \perp v_{i} & i=3,5,6 .
\end{array}
$$

A standard form for the six product vectors $w_{i}=u_{i} \otimes v_{i}$ in the range of $\rho$ is

$$
\begin{gathered}
u=\left(\begin{array}{cccccc}
1 & 0 & 0 & a_{4} & 1 & 0 \\
0 & 1 & 0 & 1 & 0 & b_{6} \\
0 & 0 & 1 & 0 & c_{5} & 1
\end{array}\right), \quad v=\left(\begin{array}{ccccccc}
0 & d_{2} & 1 & 0 & 0 & 1 \\
1 & 0 & e_{3} & 0 & 1 & 0 \\
f_{1} & 1 & 0 & 1 & 0 & 0
\end{array}\right) \\
w=\left(\begin{array}{cccccc}
0 & 0 & 0 & 0 & 0 & 0 \\
1 & 0 & 0 & 0 & 1 & 0 \\
f_{1} & 0 & 0 & a_{4} & 0 & 0 \\
0 & d_{2} & 0 & 0 & 0 & b_{6} \\
0 & 0 & 0 & 0 & 0 & 0 \\
0 & 1 & 0 & 1 & 0 & 0 \\
0 & 0 & 1 & 0 & 0 & 1 \\
0 & 0 & e_{3} & 0 & c_{5} & 0 \\
0 & 0 & 0 & 0 & 0 & 0
\end{array}\right) .
\end{gathered}
$$


Let $A$ be the $6 \times 6$ matrix that results from removing the rows of zeros, i.e. the rows one, five and nine from $w$, then

$$
\operatorname{det} A=d_{2} e_{3} f_{1}-a_{4} b_{6} c_{5}
$$

Since we want the six product vectors $w_{i}$ to span a five-dimensional space, we require that $\operatorname{det} A=0$. This equation determines the sixth parameter uniquely if we choose random values for five of the parameters $a_{4}, b_{6}, c_{5}, d_{2}, e_{3}, f_{1}$.

We impose the further restrictions that all the six parameters $a_{4}, \ldots, f_{1}$ should be nonzero, and that

$$
\left|\begin{array}{ccc}
a_{4} & 1 & 0 \\
1 & 0 & b_{6} \\
0 & c_{5} & 1
\end{array}\right|=-1-a_{4} b_{6} c_{5} \neq 0, \quad\left|\begin{array}{ccc}
0 & d_{2} & 1 \\
1 & 0 & e_{3} \\
f_{1} & 1 & 0
\end{array}\right|=1+d_{2} e_{3} f_{1} \neq 0
$$

Then, the three vectors $u_{2}, u_{3}, u_{6}$ are linearly dependent, and so are $u_{1}, u_{3}, u_{5}$ and $u_{1}, u_{2}, u_{4}$, but these are the only sets of three vectors $u_{i}$ that are linearly dependent. In the same way, the only sets of three linearly dependent vectors $v_{i}$ are $v_{1}, v_{4}, v_{5}$, then $v_{2}, v_{4}, v_{6}$ and $v_{3}, v_{5}, v_{6}$. These linear dependencies make it possible for the three product vectors $z_{1}, z_{2}, z_{3}$, and only these, to be orthogonal to all the six product vectors $w_{i}$. One may check that with these choices of parameters, there are no more product vectors that are linear combinations of the six vectors $w_{i}$.

It should be noted that with our standard form for the three product vectors $z_{i}=x_{i} \otimes y_{i}$, we still have a further freedom of doing diagonal product transformations. In this way, we may actually reduce the number of parameters in our standard form from six to one, setting for example, $a_{4}=c_{5}=d_{2}=f_{1}=1$. We must then set $b_{6}=e_{3} \neq 0,-1$ in order to satisfy all the conditions. Setting $b_{6}=e_{3}=1 / p$, we then get

$$
u=\left(\begin{array}{llllll}
1 & 0 & 0 & 1 & 1 & 0 \\
0 & 1 & 0 & 1 & 0 & 1 \\
0 & 0 & 1 & 0 & 1 & p
\end{array}\right), \quad v=\left(\begin{array}{llllll}
0 & 1 & p & 0 & 0 & 1 \\
1 & 0 & 1 & 0 & 1 & 0 \\
1 & 1 & 0 & 1 & 0 & 0
\end{array}\right)
$$

A PPT state $\rho$ with $\operatorname{Img} \rho$ spanned by the product vectors $w_{1}, \ldots, w_{6}$ and with $z_{1}, z_{2}, z_{3} \in \operatorname{Ker} \rho$ must also have $z_{1}, z_{2}, z_{3} \in \operatorname{Ker} \rho^{P}$ since $y_{1}, y_{2}, y_{3}$ are real. For given $w$ and $z$, these restrictions on $\rho$ reduce our search for such states to a seven-dimensional subspace of $H_{9}$. Six of the seven dimensions are spanned by the pure product states $\rho_{i}=N_{i} w_{i} w_{i}^{\dagger}$, with normalization factors $N_{i}$, and there is only one dimension left where there is room for entangled PPT states. With $u$ and $v$ defined as in 
Eq. (71), the seventh direction is given by the matrix

$$
\sigma=\left(\begin{array}{rrrrrrrrr}
0 & 0 & 0 & 0 & 0 & 0 & 0 & 0 & 0 \\
0 & 0 & -\mathrm{i} & 0 & 0 & 0 & 0 & \mathrm{i} & 0 \\
0 & \mathrm{i} & 0 & 0 & 0 & -\mathrm{i} & 0 & 0 & 0 \\
0 & 0 & 0 & 0 & 0 & \mathrm{i} & -\mathrm{i} p^{*} & 0 & 0 \\
0 & 0 & 0 & 0 & 0 & 0 & 0 & 0 & 0 \\
0 & 0 & \mathrm{i} & -\mathrm{i} & 0 & 0 & 0 & 0 & 0 \\
0 & 0 & 0 & \mathrm{i} p & 0 & 0 & 0 & -\mathrm{i} p & 0 \\
0 & -\mathrm{i} & 0 & 0 & 0 & 0 & \mathrm{i} p^{*} & 0 & 0 \\
0 & 0 & 0 & 0 & 0 & 0 & 0 & 0 & 0
\end{array}\right) .
$$

In random numerical searches, we have produced different sets of six product vectors and hundreds of rank $(5,5)$ PPT states in the corresponding seven-dimensional subspace of $H_{9}$. All the states we find are entangled but not extremal. The only extremal PPT states in this subspace are the six pure product states $\rho_{i}$ and a fourdimensional surface of rank $(4,4)$ states. The states $\rho_{i}$ define a five-dimensional simplex of separable states, which contain two special equilateral triangles with corners $\rho_{1}, \rho_{2}, \rho_{3}$ and $\rho_{4}, \rho_{5}, \rho_{6}$.

A convex combination of an arbitrary rank $(4,4)$ extremal PPT state and an arbitrary separable state from one of the two special triangles is always a rank $(5,5)$ entangled PPT state and every rank $(5,5)$ PPT state of the above form can be constructed in this way. Thus, it lies inside a three-dimensional set of rank $(5,5) \mathrm{PPT}$ states bounded by an equilateral triangle, either $\rho_{1}, \rho_{2}, \rho_{3}$ or $\rho_{4}, \rho_{5}, \rho_{6}$, and a twodimensional surface of extremal rank $(4,4)$ PPT states. An example of this geometry is shown in Fig. 2. It implies that when $\rho$ is a rank $(5,5)$ PPT entangled state, there are always three product vectors $w_{i}=u_{i} \otimes v_{i} \in \operatorname{Img} \rho$ such that $\tilde{w}_{i}=$ $u_{i} \otimes v_{i}^{*} \in \operatorname{Img} \rho^{P}$. The two possible sets of three are $i=1,2,3$ or $i=4,5,6$. Since there are less than five such product vectors, the range criterion is not fulfilled.

A separable state mixed from $k$ product states $\rho_{i}$ has rank $(k, k)$ if $k<6$. It has rank $(5,6)$ and lies in the interior of the simplex if $k=6$. Rank $(5,6)$ entangled states arise in many ways, for example, as convex combinations of one rank $(4,4)$ extremal state and pure product states from both special triangles, for example, $\rho_{1}$ and $\rho_{4}$. Ranks higher than six are of course impossible since $\operatorname{Ker} \rho^{P}$ contains the three product vectors $z_{1}, z_{2}, z_{3}$. Another consequence of the last fact is that $\operatorname{Img} \rho^{P}$ contains six product vectors of the form in Eq. (68), usually with other values for the coefficients $a_{4}, b_{6}, c_{5}, d_{2}, e_{3}, f_{1}$.

We conclude that all of the rank $(5,5)$ states produced in these searches are $\{6,6 ; 3\}$ entangled nonextremal states that are neither edge states nor satisfy the range criterion. 


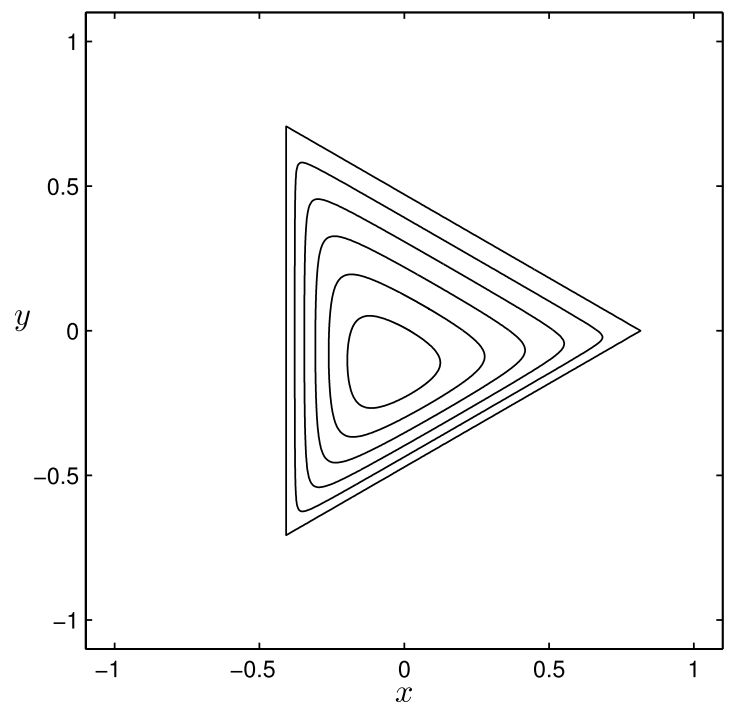

Fig. 2. A three-dimensional set, the interior of which consists of nonextremal entangled rank $(5,5)$ PPT states with $n_{\text {ker }}=3$. The set is bounded by an equilateral triangle at $z=0$ defined by the three pure product states $\rho_{4}, \rho_{5}, \rho_{6}$ and a two-dimensional surface with $z>0$ of extremal rank $(4,4)$ PPT states. The curved lines are equidistant contours at intervals of $\Delta z=0.07$. The coordinates $x, y, z$ are dimensionless.

\section{Case IV: $n_{\text {ker }}=2$}

Given two product vectors in the kernel of $\rho$, we can always make a product transformation so that we get for these $z_{i}=x_{i} \otimes y_{i}$ for $i=1,2$ from Eq. (45).

We have the freedom of doing further product transformations with

$$
\left(V_{A}^{\dagger}\right)^{-1} x_{i}=\alpha_{i} x_{i}, \quad\left(V_{B}^{\dagger}\right)^{-1} y_{i}=\beta_{i} y_{i}, \quad \alpha_{i}, \beta_{i} \in \mathbb{C}, \quad i=1,2 .
$$

In order to have six product vectors $w_{i}=u_{i} \otimes v_{i}$ orthogonal to $z_{1}, z_{2}$, one possibility is to impose the following orthogonality conditions:

$$
\begin{aligned}
& x_{1} \perp u_{i} \quad i=1,2,3, \quad y_{1} \perp v_{i} \quad i=4,5,6, \\
& x_{2} \perp u_{i} \quad i=3,4,5, \quad y_{2} \perp v_{i} \quad i=1,2,6 \text {. }
\end{aligned}
$$

These conditions are satisfied when the six product vectors $w_{i}=u_{i} \otimes v_{i}$ in the range of $\rho$ have the standard form

$$
u=\left(\begin{array}{cccccc}
0 & 0 & 0 & a_{4} & a_{5} & a_{6} \\
b_{1} & b_{2} & 0 & 0 & 0 & b_{6} \\
1 & 1 & 1 & 1 & 1 & 1
\end{array}\right), \quad v=\left(\begin{array}{cccccc}
d_{1} & d_{2} & d_{3} & 0 & 0 & 0 \\
0 & 0 & e_{3} & e_{4} & e_{5} & 0 \\
1 & 1 & 1 & 1 & 1 & 1
\end{array}\right)
$$


with $a_{i}, b_{i}, d_{i}, e_{i} \in \mathbb{C}$. We then get

$$
w=\left(\begin{array}{cccccc}
0 & 0 & 0 & 0 & 0 & 0 \\
0 & 0 & 0 & a_{4} e_{4} & a_{5} e_{5} & 0 \\
0 & 0 & 0 & a_{4} & a_{5} & a_{6} \\
b_{1} d_{1} & b_{2} d_{2} & 0 & 0 & 0 & 0 \\
0 & 0 & 0 & 0 & 0 & 0 \\
b_{1} & b_{2} & 0 & 0 & 0 & b_{6} \\
d_{1} & d_{2} & d_{3} & 0 & 0 & 0 \\
0 & 0 & e_{3} & e_{4} & e_{5} & 0 \\
1 & 1 & 1 & 1 & 1 & 1
\end{array}\right) .
$$

We may generate random values of the coefficients $a_{i}, b_{i}, d_{i}, e_{i}$ defining any five of the six product vectors $w_{i}$. Then, we always find a sixth product vector, unique up to normalization, which is a linear combination of the five. The complete set of six product vectors then has rank five and automatically takes the form given in Eq. (76). It is straightforward to check that $z_{1}, z_{2}$ are the only product vectors orthogonal to all the $w_{i}$.

We generate rank $(5,5)$ PPT states $\rho$ with $w_{1}, \ldots, w_{6} \in \operatorname{Img} \rho$. With the additional restriction that the two fixed product vectors $z_{1}$ and $z_{2}$ should be in $\operatorname{Ker} \rho^{P}$, the search can be restricted to an 11-dimensional subspace of the 81-dimensional space $H_{9}$ of Hermitian matrices.

With different sets of product vectors generated in this way, we have produced numerically, by random searches, hundreds of rank $(5,5)$ PPT states. They are all extremal. Hence, they are also edge states, that is, when $u_{i} \otimes v_{i} \in \operatorname{Img} \rho$, we never have $u_{i} \otimes v_{i}^{*} \in \operatorname{Img} \rho^{P}$. The partial transpose $\rho^{P}$ of such a state $\rho$ always has the same characteristics, i.e. there are six product vectors in $\operatorname{Img} \rho^{P}$ of the form given in Eq. (76), but with different values of the coefficients $a_{i}, b_{i}, d_{i}, e_{i}$.

In summary, all the states produced in this way are $\{6,6 ; 2\}$ extremal states.

\section{Case VII: $n_{\text {ker }}=2$ with symmetric orthogonality relations}

Observe that for the above standard form given in Eq. (75), the orthogonality relations in Eq. (74) overlap. This has been done intentionally and the reason will become clear when we now try to impose the more symmetric orthogonality relations

$$
\begin{array}{llll}
x_{1} \perp u_{i} & i=1,2,3, & y_{1} \perp v_{i} & i=4,5,6, \\
x_{2} \perp u_{i} & i=4,5,6, & y_{2} \perp v_{i} & i=1,2,3 .
\end{array}
$$

This would give six product vectors $w_{i}=u_{i} \otimes v_{i}$ in $\operatorname{Img} \rho$ on a standard form

$$
u=\left(\begin{array}{cccccc}
0 & 0 & 0 & a_{4} & a_{5} & a_{6} \\
b_{1} & b_{2} & b_{3} & 0 & 0 & 0 \\
1 & 1 & 1 & 1 & 1 & 1
\end{array}\right), \quad v=\left(\begin{array}{cccccc}
d_{1} & d_{2} & d_{3} & 0 & 0 & 0 \\
0 & 0 & 0 & e_{4} & e_{5} & e_{6} \\
1 & 1 & 1 & 1 & 1 & 1
\end{array}\right)
$$


where $a_{i}, b_{i}, d_{i}, e_{i} \in \mathbb{C}$. As compared to Eq. (83), it means that we set $b_{4}=b_{5}=b_{6}=e_{1}=e_{2}=e_{3}=0$. We then get

$$
w=\left(\begin{array}{cccccc}
0 & 0 & 0 & 0 & 0 & 0 \\
0 & 0 & 0 & a_{4} e_{4} & a_{5} e_{5} & a_{6} e_{6} \\
0 & 0 & 0 & a_{4} & a_{5} & a_{6} \\
b_{1} d_{1} & b_{2} d_{2} & b_{3} d_{3} & 0 & 0 & 0 \\
0 & 0 & 0 & 0 & 0 & 0 \\
b_{1} & b_{2} & b_{3} & 0 & 0 & 0 \\
d_{1} & d_{2} & d_{3} & 0 & 0 & 0 \\
0 & 0 & 0 & e_{4} & e_{5} & e_{6} \\
1 & 1 & 1 & 1 & 1 & 1
\end{array}\right) .
$$

Omitting one of the six product vectors gives rank $w=5$. However, omitting for example $w_{6}$, we can find no sixth product vector of the form $w_{6}$ in the fivedimensional subspace. On the other hand, there will be infinitely many product vectors that are linear combinations of $w_{1}, w_{2}, w_{3}$. In fact, $u_{3}$ and $v_{3}$ must be linear combinations

$$
u_{3}=\alpha_{1} u_{1}+\alpha_{2} u_{2}, \quad v_{3}=\beta_{1} v_{1}+\beta_{2} v_{2},
$$

and then for any $\gamma_{1}, \gamma_{2} \in \mathbb{C}$, the product vector $w^{\prime}=u^{\prime} \otimes v^{\prime}$ with

$$
u^{\prime}=\gamma_{1} \alpha_{1} u_{1}+\gamma_{2} \alpha_{2} u_{2}, \quad v^{\prime}=\gamma_{1} \beta_{1} v_{1}+\gamma_{2} \beta_{2} v_{2}
$$

will be a linear combination of $w_{1}, w_{2}, w_{3}$. All these product vectors lie in a three-dimensional subspace of a $\mathbb{C}^{2} \otimes \mathbb{C}^{2}$ subspace.

Our search for $\operatorname{rank}(5,5) \operatorname{PPT}$ states $\rho$ with $w_{1}, \ldots, w_{5} \in \operatorname{Img} \rho$ and $z_{1}, z_{2} \in$ Ker $\rho^{P}$ can now be restricted to a 13-dimensional subspace of $H_{9}$. Using five such randomly generated product vectors $w_{1}, \ldots, w_{5}$ to create subspaces of dimension five, we have produced numerically by random searches hundreds of rank $(5,5)$ PPT states. Most of the states we find are separable, but we also find a very small number of extremal rank $(5,5)$ PPT states.

We find that 11 of the 13 dimensions in $H_{9}$ represent unnormalized separable states, and the entangled PPT states account for the last two of the 13 dimensions. This can be understood as follows. The product vectors $w_{1}, w_{2}, w_{3}$ span a threedimensional subspace containing infinitely many product vectors. These product vectors generate a set of unnormalized separable states of dimension nine, the same as the complete set of unnormalized density matrices on the three-dimensional subspace. In addition, we get two more dimensions of separable states from the product vectors $w_{4}$ and $w_{5}$.

Note that a separable state mixed from more than three product vectors in the $\mathbb{C}^{2} \otimes \mathbb{C}^{2}$ subspace will have rank $(3,4)$. Hence, if we also mix in one or both of $w_{4}, w_{5}$, we will get separable states of rank $(4,5)$ or rank $(5,6)$, respectively. 
The partial transposes of the states we construct numerically have the same characteristics, thus all the states are of type $\{\infty, \infty ; 2\}$. It is noteworthy that it is possible for an extremal rank $(5,5) \mathrm{PPT}$ state to have infinitely many product vectors in its range.

\section{Case II: $n_{\text {ker }}=\mathbf{1}$}

Given a product vector in the kernel of $\rho$, we may perform a product transformation as in Eq. (31) so that the vector after normalization takes the form $z_{1}=x_{1} \otimes y_{1}$ from Eq. (45).

This transformation is not unique, and we have the freedom of doing further transformations with

$$
\left(V_{A}^{\dagger}\right)^{-1} x_{1}=\alpha x_{1}, \quad\left(V_{B}^{\dagger}\right)^{-1} y_{1}=\beta y_{1}, \quad \alpha, \beta \in \mathbb{C} .
$$

We assume that the six product vectors $w_{i}=u_{i} \otimes v_{i}$ in the range of $\rho$ have the standard form

$$
u=\left(\begin{array}{cccccc}
0 & 0 & 0 & a_{4} & a_{5} & a_{6} \\
b_{1} & b_{2} & b_{3} & b_{4} & b_{5} & b_{6} \\
1 & 1 & 1 & 1 & 1 & 1
\end{array}\right), \quad v=\left(\begin{array}{cccccc}
d_{1} & d_{2} & d_{3} & 0 & 0 & 0 \\
e_{1} & e_{2} & e_{3} & e_{4} & e_{5} & e_{6} \\
1 & 1 & 1 & 1 & 1 & 1
\end{array}\right)
$$

where $a_{i}, b_{i}, d_{i}, e_{i} \in \mathbb{C}$. They are then orthogonal to $z_{1}=x_{1} \otimes y_{1}$ because

$$
x_{1} \perp u_{i} \quad i=1,2,3, \quad y_{1} \perp v_{i} \quad i=4,5,6 .
$$

The normalization in Eq. (83) presupposes that the third component of every vector is nonzero. There is no loss of generality in this assumption because of the freedom to do product transformations. This freedom could actually be used to reduce the number of parameters in the standard form given in Eq. (83).

In our numerical searches we generate, for example, random values for the coefficients $a_{i}, b_{i}, d_{i}, e_{i}$ for $i=1, \ldots, 5$. This is then a generic case in which $w_{1}, \ldots, w_{5}$ span a five-dimensional subspace, and there will be a unique solution for $a_{6}, b_{6}, e_{6}$ such that $w_{6}$ lies in this subspace. There are no other product vectors in the subspace in the generic case.

Furthermore, there can be no product vector $z^{\prime}=x^{\prime} \otimes y^{\prime}$ orthogonal to all $w_{i}$, apart from $z^{\prime}=z_{1}$. In fact, $x^{\prime}$ can at most be orthogonal to three vectors $u_{i}$ because any four of the vectors $u_{i}$ span $\mathbb{C}^{3}$. Similarly, $y^{\prime}$ can at most be orthogonal to three vectors $v_{i}$. The only set of three linearly dependent vectors $u_{i}$ is $u_{1}, u_{2}, u_{3}$, and the only set of three linearly dependent vectors $v_{i}$ is $v_{4}, v_{5}, v_{6}$. The only possibility is therefore that $x^{\prime} \perp u_{i}$ with $i=1,2,3$ and $y^{\prime} \perp v_{i}$ with $i=4,5,6$, implying that $z^{\prime}=z_{1}$. 
We generate rank $(5,5) \mathrm{PPT}$ states with this subspace as range. In general, there is a 25-dimensional real vector space of Hermitian matrices operating on a fixed subspace of dimension five. However, when we are searching for a PPT state $\rho$ with $\rho z_{1}=0$, we have the additional restriction that the partially conjugated product vector $\tilde{z}_{1}=x_{1} \otimes y_{1}^{*}=x_{1} \otimes y_{1}=z_{1}$ must be in Ker $\rho^{P}$. This reduces the 25 dimensions to 17 because the equation $\rho^{P} z_{1}=0$ gives the four extra complex equations $\rho_{j k}=0$ with $j=2,3$ and $k=4,7$. It is straightforward to find numerically rank $(5,5)$ PPT states in this 17-dimensional space of Hermitian matrices.

In random searches, we have produced hundreds of such states for many different sets of product vectors $w_{i}$. All states produced in this manner are extremal. Hence, they are edge states, that is, when $u_{i} \otimes v_{i} \in \operatorname{Img} \rho$, we never have $u_{i} \otimes v_{i}^{*} \in \operatorname{Img} \rho^{P}$. We find that the partial transpose $\rho^{P}$ always has the same characteristics as $\rho$, i.e. there are six product vectors in $\operatorname{Img} \rho^{P}$ of the form in Eq. (83), but with different values for the coefficients $a_{i}, b_{i}, d_{i}, e_{i}$.

So, in our classification, all these states are $\{6,6 ; 1\}$ extremal states.

\section{Case III: An Exceptional Subspace of Type $\{2 ; 1\}$}

A generic five-dimensional subspace of $\mathbb{C}^{3} \otimes \mathbb{C}^{3}$ contains exactly six product vectors. Obviously, a nongeneric five-dimensional subspace may well contain infinitely many product vectors if it contains a whole product space $\mathbb{C}^{1} \otimes \mathbb{C}^{2}, \mathbb{C}^{2} \otimes \mathbb{C}^{1}$ or $\mathbb{C}^{2} \otimes \mathbb{C}^{2}$. On the other hand, it may also contain less than six product vectors if the set of equations in (15) have degenerate solutions. ${ }^{11}$ The maximum dimension of an entangled subspace, i.e. a subspace that does not contain any product vectors, is known from Ref. 15 , and for the $3 \times 3$ system, the limiting dimension is four. So, any subspace of $\mathbb{C}^{9}$ of dimension five or higher must contain at least one product vector. We describe here a very special $\{2 ; 1\}$ subspace of $\mathbb{C}^{9}$.

In our random searches for rank $(5,5) \mathrm{PPT}$ states with the special symmetry property that the state $\rho$ and its partial transpose $\rho^{P}$ are SL $\otimes$ SL-equivalent, we have found four such states with $n_{\text {img }}=2$. A further special property of these states is that $n_{\text {ker }}=1$, i.e. there is exactly one product vector in $\operatorname{Ker} \rho$. The states are not extremal, since three of them lie on a line between a pure product state and a rank $(4,4)$ extremal PPT state, whereas one lies inside a two-dimensional region bounded by a closed curve of rank $(4,4)$ extremal PPT states.

Further numerical random searches for rank $(5,5)$ PPT states with the same ranges as the nonextremal rank $(5,5) \mathrm{PPT}$ states described above, but not restricted to $\mathrm{SL} \otimes \mathrm{SL}-$ symmetric states, reveal extremal rank $(5,5)$ PPT states of type $\{2,6 ; 1\}$. It is clear that a subspace with only two product vectors supports just one line of separable states.

We find that the pure product state and the curve of rank $(4,4)$ states are parts of the same geometry, which can be transformed to a standard form as shown in Fig. 3. It is an empirical fact that a rank $(4,4)$ state $\rho_{44}$ involved in such a construction of a 


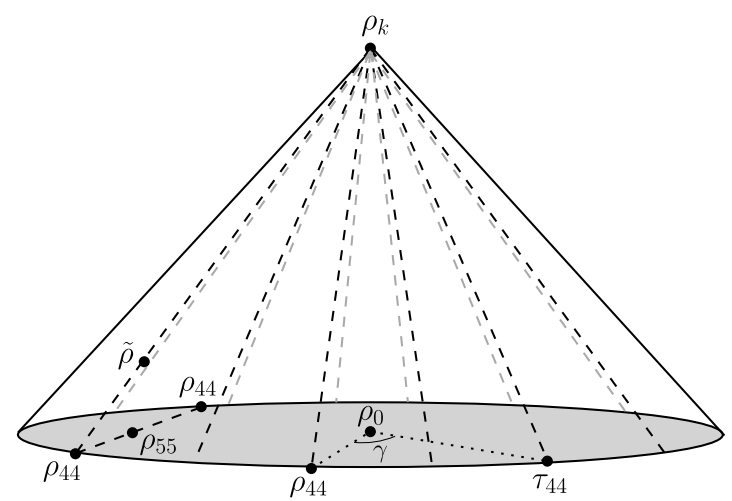

Fig. 3. A pure product state $\rho_{k}=w_{k} w_{k}^{\dagger}{ }^{\dagger}$ with $k=1$ or $k=2$, together with the circle of extremal rank $(4,4)$ PPT states, defines a three-dimensional conical face of $\mathcal{P}$. The state $\rho_{0}$ is defined as the center of the circle.

rank $(5,5)$ state $\rho$ can always be transformed to a standard form where the product vectors in Ker $\rho_{44}$ are related to regular icosahedra. ${ }^{19}$ We will now describe this standard form in more detail.

\subsection{Rank $(5,5)$ PPT states in a region bounded by rank $(4,4)$ PPT states}

We describe here analytically a set of states found by transformation to standard form of one particular rank $(5,5)$ nonextremal PPT state found numerically in a random search. This particular state lies inside a curve of rank $(4,4)$ states, but the three other states we have found belong to the same geometry. It is remarkable that analytically defined states possessing such very special properties turn up in random searches. Our limited imagination would not have enabled us to deduce their existence.

After transformation to standard form, the rank $(5,5)$ state lies inside a circle bounded by extremal rank $(4,4)$ PPT states. The interior of the circle consists entirely of rank $(5,5)$ PPT states, each of which has exactly one product vector in its kernel, this vector is common to all the rank $(4,4)$ and rank $(5,5)$ states. Each of the rank $(4,4)$ states has five additional product vectors in its kernel, these are different for the different states. The rank $(4,4)$ states have no product vectors in their ranges, whereas all the rank $(5,5)$ states have one common range containing exactly two product vectors. All the rank $(4,4)$ and rank $(5,5)$ states are symmetric under partial transposition.

\section{Product vectors from a regular icosahedron}

In the standard form we define, all the product vectors in the kernels of all the rank $(4,4)$ states are defined from regular icosahedra, as follows. We define 
$c_{k}=\cos (2 k \pi / 5), s_{k}=\sin (2 k \pi / 5)$, thus

$$
\begin{array}{ll}
c_{1}=c_{4}=\frac{\sqrt{5}-1}{4}, & s_{1}=-s_{4}=\frac{\sqrt{10+2 \sqrt{5}}}{4}, \\
c_{2}=c_{3}=-\frac{\sqrt{5}+1}{4}, & s_{2}=-s_{3}=\frac{\sqrt{10-2 \sqrt{5}}}{4} .
\end{array}
$$

Note that $\phi=2 c_{1}=0.61803 \ldots$ is the golden mean, defined by the equation $\phi^{2}=1-\phi$.

We define product vectors $z_{k}=x_{k} \otimes y_{k}$ for $k=1,2, \ldots, 6$ with

$$
x=\frac{1}{\sqrt{5}}\left(\begin{array}{cccccc}
2 & 2 c_{1} & 2 c_{2} & 2 c_{3} & 2 c_{4} & 0 \\
0 & 2 s_{1} & 2 s_{2} & 2 s_{3} & 2 s_{4} & 0 \\
1 & 1 & 1 & 1 & 1 & \sqrt{5}
\end{array}\right), \quad y=\frac{1}{\sqrt{5}}\left(\begin{array}{cccccc}
2 c_{2} & 2 & 2 c_{3} & 2 c_{1} & 2 c_{4} & 0 \\
2 s_{2} & 0 & 2 s_{3} & 2 s_{1} & 2 s_{4} & 0 \\
1 & 1 & 1 & 1 & 1 & \sqrt{5}
\end{array}\right) \text {. }
$$

The 12 vectors $\pm x_{k}$ are real and are all the corners of a regular icosahedron. The vectors $y_{k}$ as defined here are the same vectors in a different order. We define $x$ and $y$ in such a way that $x_{5}=y_{5}$ and $x_{6}=y_{6}$. The product vector $z_{6}$ is going to play a special role, and it will be the one common product vector in the kernels of any two of the rank $(4,4)$ states.

\section{A circle of extremal rank $(4,4)$ PPT states}

The six product vectors $z_{k}$ are linearly dependent and define a five-dimensional subspace of $\mathbb{C}^{9}$. The orthogonal projection on this subspace may be written as

$$
Q=\frac{5}{6} \sum_{k=1}^{6} z_{k} z_{k}^{\dagger} .
$$

Since $Q$ is a projection, with $Q^{2}=Q$, and is symmetric under partial transposition, $Q^{P}=Q$, we conclude that the matrix

$$
\tau_{44}=\frac{1}{4}(I-Q)
$$

is a rank $(4,4)$ PPT state, entangled and extremal. This method for constructing such states is known as a UPB construction, since any five of the six vectors $z_{k}$ form a UPB for the subspace, so-called because there is no product vector orthogonal to all of them..$^{8,9}$

Note that $Q$ and $\tau_{44}$ are symmetric under partial transposition with respect to subsystem $B$, in our notation $Q^{P}=Q$ and $\tau_{44}^{P}=\tau_{44}$, because the vectors $y_{k}$ defined in Eq. (86) are real. They are also symmetric under partial transposition with respect to subsystem $A$, in our notation $Q^{P T}=Q$ and $\tau_{44}^{P T}=\tau_{44}$, because the vectors $x_{k}$ are real. 


\section{O. Hansen $\mathcal{E}$ J. Myrheim}

We now define product vectors $w_{k}=u_{k} \otimes v_{k}$ for $k=1,2$ with

$$
u_{1}=\frac{1}{\sqrt{2}}\left(\begin{array}{c}
1 \\
-\mathrm{i} \\
0
\end{array}\right), \quad u_{2}=\left(\begin{array}{l}
0 \\
0 \\
1
\end{array}\right), \quad v_{1}=v_{2}^{*}=u_{1}^{*} .
$$

Note that $u_{2}=x_{6}=y_{6}$ and

$$
u_{1}=\frac{1}{\sqrt{10}} \sum_{j=1}^{5} \omega^{j-1} x_{j}
$$

with $\omega=\mathrm{e}^{-2 \pi \mathrm{i} / 5}=c_{1}-\mathrm{i} s_{1}$.

We define

$$
W=w_{1} w_{2}^{\dagger}=\left(u_{1} u_{2}^{\dagger}\right) \otimes\left(v_{1} v_{2}^{\dagger}\right)
$$

and then the two Hermitian matrices

$$
A=W+W^{\dagger}, \quad B=\mathrm{i}\left(W-W^{\dagger}\right) .
$$

Since $v_{1}=v_{2}^{*}, W$ is symmetric under partial transposition,

$$
W=\left(u_{1} u_{2}^{\dagger}\right) \otimes\left(v_{1} v_{1}^{T}\right)=\left(u_{1} u_{2}^{\dagger}\right) \otimes\left(v_{1} v_{1}^{T}\right)^{T}=W^{P}
$$

and so are $A$ and $B$. Define now

$$
\rho=\tau_{44}+\alpha A+\beta B
$$

with real parameters $\alpha$ and $\beta$. The eigenvalues of $\rho$ are four times 0 , three times $1 / 4$ and

$$
\lambda_{ \pm}=\frac{1}{8} \pm \frac{1}{24} \sqrt{1+\left(24 \alpha-2 \sqrt{2} c_{1}\right)^{2}+\left(24 \beta-2 \sqrt{2} s_{1}\right)^{2}}
$$

Since $\rho^{P}=\rho$, we get a rank $(4,4)$ PPT state with $\lambda_{-}=0$ when

$$
\left(24 \alpha-2 \sqrt{2} c_{1}\right)^{2}+\left(24 \beta-2 \sqrt{2} s_{1}\right)^{2}=8 .
$$

In particular, $\alpha=\beta=0$ gives the state $\tau_{44}$ that we started from. Equation (96) defines a circle of rank $(4,4)$ states $\rho_{44}$, which are in fact extremal PPT states. All the states inside the circle are rank $(5,5)$ nonextremal PPT states. The center of the circle we call $\rho_{0}$, it has $\alpha=\sqrt{2} c_{1} / 12$ and $\beta=\sqrt{2} s_{1} / 12$.

All the states defined by Eq. (94) are symmetric under partial transposition with respect to subsystem $B$, we have that

$$
\rho^{P}=\tau_{44}^{P}+\alpha A^{P}+\beta B^{P}=\tau_{44}+\alpha A+\beta B=\rho .
$$

However, because $A$ and $B$ are complex, $\rho$ is complex when $\alpha \neq 0$ or $\beta \neq 0$. Then, it is not symmetric under partial transposition with respect to subsystem $A$, we have then

$$
\rho^{P T}=\rho^{T}=\rho^{*} \neq \rho .
$$

All the rank $(4,4)$ states $\rho_{44}$ on the circle are projections. The rank $(4,4)$ state at an angle $\gamma$ around the circle from the state $\tau_{44}$ is $\rho_{44}=U \tau_{44} U^{\dagger}$, where $U=U_{A} \otimes U_{B}$ 
is a unitary product transformation,

$$
\begin{gathered}
U_{A}=\left(\begin{array}{ccc}
\cos (\gamma / 10) & -\sin (\gamma / 10) & 0 \\
\sin (\gamma / 10) & \cos (\gamma / 10) & 0 \\
0 & 0 & \mathrm{e}^{-\mathrm{i} \gamma / 2}
\end{array}\right), \\
U_{B}=\left(\begin{array}{ccc}
\cos (\gamma / 5) & \sin (\gamma / 5) & 0 \\
-\sin (\gamma / 5) & \cos (\gamma / 5) & 0 \\
0 & 0 & 1
\end{array}\right) .
\end{gathered}
$$

The kernel of $U \tau_{44} U^{\dagger}$ is defined by the transformed icosahedron vectors $\left(U_{A}^{\dagger}\right)^{-1} x=$ $U_{A} x$ and $\left(U_{B}^{\dagger}\right)^{-1} y=U_{B} y$. This transformation leaves $y_{6}$ invariant and $x_{6}$ invariant up to a phase factor. A rotation by $\gamma=2 \pi$ is a cyclic permutation of the first five icosahedron vectors,

$$
x_{1} \mapsto-x_{4} \mapsto x_{2} \mapsto-x_{5} \mapsto x_{3} \mapsto-x_{1},
$$

and

$$
y_{1} \mapsto y_{4} \mapsto y_{2} \mapsto y_{5} \mapsto y_{3} \mapsto y_{1}
$$

Note that the vectors $u_{1}, u_{2}, v_{1}$ and $v_{2}$ are eigenvectors of the transformation matrices $U_{A}$ and $U_{B}$, for example,

$$
U_{A} u_{1}=\mathrm{e}^{\mathrm{i} \gamma / 10} u_{1} .
$$

Hence, the pure states $\rho_{k}=w_{k} w_{k}^{\dagger}$ are invariant under the transformation $\rho \mapsto U \rho U^{\dagger}$.

\section{Two three-dimensional solid cones}

The geometry of this construction is depicted in Fig. 3. The four traceless matrices $A, B, \rho_{1}-\rho_{0}$ and $\rho_{2}-\rho_{0}$ define directions in $H_{9}$ that are mutually orthogonal. The directions $A$ and $B$ define the plane of the circle centered on $\rho_{0}$. Thus, the two states $\rho_{k}=w_{k} w_{k}^{\dagger}$ with $k=1,2$, together with the circle of rank $(4,4)$ states, define two cones that are different three-dimensional faces of $\mathcal{P}$, the set of PPT states. All the nonextremal states on the surface of a cone are rank $(5,5)$ states. A state $\rho$ in the interior of the cone may be written as a convex combination

$$
\rho=p \rho_{55}+(1-p) \rho_{k}, \quad 0<p<1
$$

with $\rho_{55}$ inside the circle and $k=1,2$. This state also has rank five since $w_{k} \in \operatorname{Img} \rho_{55}$. But its partial transpose $\rho^{P}$ will have rank six because it is a convex combination of $\rho_{55}^{P}=\rho_{55}$ and one of the two pure product states

$$
\rho_{1}^{P}=\left(u_{1} \otimes v_{2}\right)\left(u_{1} \otimes v_{2}\right)^{\dagger}, \quad \rho_{2}^{P}=\left(u_{2} \otimes v_{1}\right)\left(u_{2} \otimes v_{1}\right)^{\dagger} .
$$

Since $\rho_{55}^{P T}=\rho_{55}^{T} \neq \rho_{55}$, that is, $\rho_{55}$ is not symmetric under partial transposition with respect to system $A$, we conclude that neither is $\rho$. The same conclusion, that 
$\rho^{P T} \neq \rho$, follows because $\rho^{P T}$ must have rank six, in fact, it has the same eigenvalues as $\rho^{P}$.

\section{The conical surface}

Since $u_{2}$ as defined in Eq. (89) is real, it follows that $\rho^{P T}=\rho$ when we define

$$
\rho=p \tau_{44}+(1-p) \rho_{2}, \quad 0<p<1 .
$$

Thus, a state $\rho$ of this form is a rank $(5,5)$ state on the surface of the cone that is symmetric under partial transposition with respect to system $A$. Since $\rho^{P}$ and $\rho^{P T}$ have the same eigenvalues, it follows that $\rho$ and $\rho^{P}$ have the same eigenvalues, although they are neither $\mathrm{SU} \otimes \mathrm{SU}$-equivalent nor $\mathrm{SL} \otimes \mathrm{SL}$-equivalent.

If we define

$$
\tilde{\rho}=p \rho_{44}+(1-p) \rho_{2},
$$

using a different rank $(4,4)$ state $\rho_{44}$ in place of the special state $\tau_{44}$, then we get a state $\tilde{\rho}$ that has no longer the same symmetry as $\rho$, but is equivalent to $\rho$ by a unitary product transformation $U$ as given in Eq. (99). In this case, again $\tilde{\rho}, \tilde{\rho}^{P}$, and $\tilde{\rho}^{P T}$ all have the same eigenvalues, in fact, they have the same eigenvalues as $\rho, \rho^{P}$ and $\rho^{P T}$.

If we replace $\rho_{2}$ by $\rho_{1}$ in Eq. (104), then the state $\rho$ we get is not $\mathrm{SL} \otimes$ SL-equivalent to any one of $\rho^{P}$ or $\rho^{P T}$. We find empirically that all three states $\rho, \rho^{P}$ and $\rho^{P T}$ still have the same set of eigenvalues, although the reason is not clear.

\section{Four similar constructions}

The construction just described is one of four different ways of extending the fourdimensional subspace Img $\tau_{44}$ to a five-dimensional subspace which is orthogonal to $z_{6}$ and contains exactly two product vectors $w_{1}, w_{2}$. Define

$$
a=\left(\begin{array}{l}
0 \\
0 \\
1
\end{array}\right), \quad b=\frac{1}{\sqrt{2}}\left(\begin{array}{l}
1 \\
\mathrm{i} \\
0
\end{array}\right) \text {. }
$$

We find that the only possibilities are those given in Table 4 . They give rise to four different circles of rank $(4,4)$ extremal PPT states. The specific possibility given by

Table 4. The four ways to extend the four-dimensional subspace $\operatorname{Img} \tau_{44}$ to a five-dimensional subspace orthogonal to $z_{6}$ and containing exactly two product vectors $w_{1}, w_{2}$.

\begin{tabular}{ccc}
\hline Case & $w_{1}$ & $w_{2}$ \\
\hline 1 & $b \otimes b^{*}$ & $a \otimes b$ \\
2 & $b^{*} \otimes b$ & $a \otimes b^{*}$ \\
3 & $b \otimes b$ & $b^{*} \otimes a$ \\
4 & $b^{*} \otimes b^{*}$ & $b \otimes a$ \\
\hline
\end{tabular}


Eq. (89) is case 2 in Table 4 . Note that the cases 1 and 2 are related by complex conjugation, and so are 3 and 4 .

In our numerical random searches for SL $\otimes$ SL-symmetric PPT states of rank $(5,5)$, we have found four examples of states that are SL $\otimes$ SL-equivalent to states lying on the surface of such a cone. In one example, the state lies inside the circle and is of the form given in Eq. (94). This is the case discussed in detail above, corresponding to case 2 in Table 4 . In the three other examples, the state lies on the conical surface and is of the form given in Eq. (104). These correspond to the cases 3 and 4 in Table 4, where $\rho_{2}^{P}=\rho_{2}$.

\section{Summary and Outlook}

The work presented here is mainly a continuation of previous studies of the entangled PPT states of rank five in the $3 \times 3$ system with an emphasis on nongeneric states.

For dimension $3 \times 3$, it is known that the extremal PPT states of lowest rank, apart from the pure product states, are entangled states of rank four. It is by now well established that all these rank four states are equivalent by SL $\otimes$ SL-transformations to states constructed by simple procedures that can be described analytically. The first such procedure, introduced by Bennett et al., was based on unextendible product bases. $^{8}$ A second procedure is described in Ref. 17. As a byproduct of our present study of rank $(5,5)$ states, we have found a third procedure, which is described here. The structure of extremal rank five PPT states is much more complex and no exhaustive classification or set of construction methods is known.

The equivalence between PPT states under SL $\otimes$ SL-transformations is an important concept, and quantities invariant under such transformations are especially useful for classification. In particular, we classify a state $\rho$ by the ranks $(m, n)$ of $\rho$ and $\rho^{P}$, and by the number of product vectors $\left\{n_{\text {img }}, \tilde{n}_{\text {ker }} ; n_{\text {img }}\right\}$ in the subspaces $\operatorname{Img} \rho$, $\operatorname{Img} \rho^{P}$ and Ker $\rho$, respectively. Generic PPT states of rank $(5,5)$ are extremal and of type $\{6,6 ; 0\}$.

As a first attempt towards a general classification, we define a state $\rho$ to be $\mathrm{SL} \otimes \mathrm{SL}$-symmetric under partial transposition if it is $\mathrm{SL} \otimes \mathrm{SL}$-equivalent to its partial transpose $\rho^{P}$. We define $\rho$ to be genuinely SL $\otimes$ SL-symmetric if it is $\mathrm{SL} \otimes \mathrm{SL}-$ equivalent to a state $\tau$ with $\tau=\tau^{P}$. We then show that genuine $\mathrm{SL} \otimes \mathrm{SL}$-symmetry implies SL $\otimes$ SL-symmetry, in such a way that at least one $\mathrm{SL} \otimes \mathrm{SL}$-transformation from $\rho$ to $\rho^{P}$ must have a special diagonal block form and in addition be trace preserving.

In random numerical searches where we searched specifically for SL $\otimes$ SL-symmetric PPT states of rank $(5,5)$, along with their associated transformations, we found about 50 such states of type $\{6,6 ; 0\}$. The condition of SL $\otimes$ SL-symmetry enforced during the search means that these states are not generic. Another restriction imposed was that we looked for product transformations that were trace preserving. This allowed us to find states that are genuinely SL $\otimes$ SL-symmetric. 
Out of the 50 states produced in this way, about half are genuinely SL $\otimes$ SL-symmetric. All these genuinely $\mathrm{SL} \otimes \mathrm{SL}$-symmetric states are $\mathrm{SL} \otimes \mathrm{SL}$-equivalent to a special class of states proposed by Chruściński and Kossakowski. ${ }^{18}$ This is a curious result which we do not understand, especially since random states of rank five that are constructed with the only restriction that they should be symmetric under partial transposition, cannot be transformed to this special form.

How to construct PPT states with $n_{\text {ker }}>0$ was discussed in Ref. 17, and we develop these matters further here. For the $3 \times 3$ system, this is essentially a study of orthogonal complementary subspaces $\mathcal{U}, \mathcal{V} \subset \mathbb{C}^{9}$ of dimensions five and four with certain nongeneric properties as described in Sec. 5.2. We have constructed several standard forms for $\mathcal{U}$ and $\mathcal{V}$, with one to four product vectors in $\mathcal{V}$, and then produced random PPT states of $\operatorname{rank}(5,5)$ with $\operatorname{Img} \rho=\mathcal{U}$ and $\operatorname{Ker} \rho=\mathcal{V}$. A detailed summary is given in Sec. 6 .

For the case $n_{\text {ker }}=4$, we find the new analytical construction, already mentioned, of all rank four extremal PPT states, up to SL $\otimes$ SL-equivalence, where they appear as boundary states on one single five-dimensional face on the set of normalized PPT states. The interior of the face consists of rank five states, a simplex of separable states surrounded by entangled PPT states. In our standard representation, all these states are real matrices, symmetric under partial transposition.

A very special subspace, of type $\{2 ; 1\}$, is collected from a small number of $\{2,2 ; 1\}$ states found in our random searches for SL $\otimes$ SL-symmetric states. We describe analytically a set of states on such a subspace, in a standard form, illustrated in Fig. 3.

Apart from this special case, we have only considered five-dimensional subspaces containing at least six product vectors. The maximum dimension of an entangled subspace, containing no product vectors, is known from Ref. 15 . For the $3 \times 3$ system, the limiting dimension is four, hence any subspace of dimension five or higher must contain at least one product vector. An analysis on how to construct subspaces with fewer than six product vectors is given in Ref. 11 . To fully describe extremal rank five PPT states according to the number of product vectors in the range and kernel, all these cases should be investigated.

Finally, we want to remind the reader that the basic problem of understanding the generic PPT states of rank $(5,5)$ remains unsolved. Also, we are even further from a full understanding of higher rank extremal PPT states in $3 \times 3$ dimensions or in higher dimensions.

\section{Acknowledgments}

We acknowledge gratefully the research grant from The Norwegian University of Science and Technology (Leif Ove Hansen) and cooperation and help from Børge Irgens especially with development of programs, but also with other insights during the research. 


\section{References}

1. J. S. Bell, Physics 1 (1964) 195.

2. A. Aspect, J. Dalibard and G. Roger, Phys. Rev. Lett. 49 (1982) 25.

3. S. Gharibian, Quantum Inf. Comput. 10 (2010) 3.

4. M. Horodecki, P. Horodecki and R. Horodecki, Phys. Lett. A 223 (1996) 1.

5. A. Peres, Phys. Rev. Lett. 77 (1996) 1413.

6. P. Horodecki, Phys. Lett. A 232 (1997) 5.

7. P. Horodecki, M. Lewenstein, G. Vidal and I. Cirac, Phys. Rev. A 62 (2000) 032310.

8. C. H. Bennett, D. P. DiVincenzo, T. Mor, P. W. Shor, J. A. Smolin and B. M. Terhal, Phys. Rev. Lett. 82 (1999) 5385.

9. D. P. DiVincenzo, T. Mor, P. W. Shor, J. A. Smolin and B. M. Terhal, Commun. Math. Phys. 238 (2003) 379.

10. J. M. Leinaas, J. Myrheim and P. Ø. Sollid, Phys. Rev. A 81 (2010) 062330.

11. L. Chen and D. Z. Djokovic, J. Math. Phys. 52 (2011) 122203.

12. J. M. Leinaas, J. Myrheim and E. Ovrum, Phys. Rev. A 76 (2007) 034304.

13. J. M. Leinaas, J. Myrheim and P. Ø. Sollid, Phys. Rev. A 81 (2010) 062329.

14. R. Hartshorne, Algebraic Geometry (Springer, New York, 2006).

15. K. R. Parthasarathy, Proc. Math. Sci. 114 (2004) 464.

16. Ł. Skowronek, J. Math. Phys. 52 (2011) 122202.

17. L. O. Hansen, A. Hauge, J. Myrheim and P. Ø. Sollid, Phys. Rev. A 85 (2012) 022309.

18. D. Chruściński and A. Kossakowski, Phys. Rev. A 76 (2007) 032308.

19. P. Ø. Sollid, J. M. Leinaas and J. Myrheim, Phys. Rev. A 84 (2011) 042325. 